Article

\title{
Optical Wireless Communication Based Indoor Positioning Algorithms: Performance Optimisation and Mathematical Modelling
}

\author{
Manisha Ajmani ${ }^{(1)} * \mathbb{D}$, Sinan Sinanović and Tuleen Boutaleb \\ School of Computing, Engineering and Built Environment, Glasgow Caledonian University, \\ 70 Cowcaddens Road, Glasgow G4 0BA, UK; sinan.sinanovic@gcu.ac.uk (S.S.); t.boutaleb@gcu.ac.uk (T.B.) \\ * Correspondence: manisha.ajmani@gcu.ac.uk; Tel.: +44-141-3313089
}

Received: 11 October 2018; Accepted: 18 December 2018; Published: 20 December 2018

\begin{abstract}
In this paper, the performance of the optimal beam radius indoor positioning (OBRIP) and two-receiver indoor positioning (TRIP) algorithms are analysed by varying system parameters in the presence of an indoor optical wireless channel modelled in line of sight configuration. From all the conducted simulations, the minimum average error value obtained for TRIP is $0.61 \mathrm{~m}$ against $0.81 \mathrm{~m}$ obtained for OBRIP for room dimensions of $10 \mathrm{~m} \times 10 \mathrm{~m} \times 3 \mathrm{~m}$. In addition, for each simulated condition, TRIP, which uses two receivers, outperforms OBRIP and reduces position estimation error up to $30 \%$. To get a better understanding of error in position estimation for different combinations of beam radius and separation between light emitting diodes, the 90th percentile error is determined using a cumulative distribution frequency (CDF) plot, which gives an error value of $0.94 \mathrm{~m}$ for TRIP as compared to $1.20 \mathrm{~m}$ obtained for OBRIP. Both algorithms also prove to be robust towards change in receiver tilting angle, thus providing flexibility in the selection of the parameters to adapt to any indoor environment. In addition, in this paper, a mathematical model based on the concept of raw moments is used to confirm the findings of the simulation results for the proposed algorithms. Using this mathematical model, closed-form expressions are derived for standard deviation of uniformly distributed points in an optical wireless communication based indoor positioning system with circular and rectangular beam shapes.
\end{abstract}

Keywords: indoor positioning system; optical wireless communication; channel modelling; position estimation; cumulative distribution frequency; standard deviation; raw moments

\section{Introduction}

Global positioning system (GPS) has revolutionised the realm of outdoor environment positioning and tracking systems [1-4], but its usability in the indoor environment is questionable $[5,6]$ owing to attenuation effects and multipath interference [3,7,8]. Radio frequency-based indoor positioning systems (IPS) suffer from electromagnetic (EM) interference $[9,10]$ and give low accuracy $[5,11]$. IPS based on radio frequency identification and ultrasound require additional infrastructure for its installation $[9,10]$, thus increasing the overall cost of the system. Optical wireless communication (OWC) with light emitting diodes (LEDs) has been touted as the ultimate solution for IPS by many researchers based on advantageous factors such as immunity to EM interference, higher accuracy as compared to other technologies, less complexity and low cost implementation [12-16]. The visible light implementation can also be simultaneously used for the purpose of illumination as well as communication [17].

OWC-based IPS can be used to track objects or people in an indoor environment. This system requires LED transmitters and optical receivers for its operation. The LED transmitters transmit their 
positional coordinates continually inside a room in the form of modulated optical signals. Upon signal reception at the receiver, positioning methods are used to decode the receiver location from the positional information of the LED transmitters [18]. The most commonly used positioning methods include triangulation, proximity or fingerprinting [19]. However, each has their own limitations [20,21] which have been discussed in the literature survey section of the paper. The principle motivation behind this research is to facilitate the design and development of a low-cost, reliable and highly accurate IPS by introducing new positioning techniques. This system can be used in hospitals and care homes, especially to better the care for people with dementia. Dementia is an ongoing illness which causes disorientation $[22,23]$ and is a serious concern for caregivers as the people with dementia may get lost and walk into dangerous situations or places. This is one of the reasons that position tracking systems can be employed to improve their care [24,25].

A major impediment in the implementation of OWC-based IPS is separation of optical signals from multiple light sources at the receiver carrying distinct positional information. Owing to recently proposed techniques aiding signal separation at the receiver, discussed in the next section, a new positioning algorithm called the optimal beam radius indoor positioning (OBRIP) algorithm is proposed in [26]. This algorithm takes the advantage of the overlapping LED light beam regions to further improve position estimation accuracy of proximity based IPS by generating more reference points for position assignment. It is designed for an indoor environment with an array of LEDs pre-installed at fixed positions continuously transmitting their location coordinates as modulated optical signals. An optical receiver attached to an object detects these signals and uses the transmitted coordinates to estimate its own location. Though the OBRIP algorithm is able to significantly improve the accuracy in position estimation, there is still scope for further improvement.

The two-receiver indoor positioning (TRIP) algorithm which builds upon the OBRIP algorithm is proposed in [27] to improve the performance of OBRIP for various system parameters. However, during their simulation the effect of propagation channel is not considered. In this paper, the OBRIP and TRIP algorithms and their corresponding setups have been simulated to analyse their performance in the presence of an indoor OWC channel in line of sight (LOS) configuration. Depending on the important channel parameter values including semi-angle at half power, field of view and physical area of detector, along with the geometrical position of the LED array and the receiver object, the impulse response at the receiver is determined which in turn helps find out the average error in position estimation. The result of the comparison between the geometrical simulation with the channel-incorporated simulation confirms the viability of the proposed algorithms in practical scenarios. In this paper, the performance of these algorithms is analysed in terms of root mean square error (RMSE), which is referred to as average error in this paper.

Mathematical validation of the results is the best way to confirm the findings of the simulations and it also helps in determining the optimal value of beam radius for minimum positioning error. Beam radius, is defined as the maximum extent up to which an LED light source can transmit with intensity greater than receiver threshold. In this paper, a novel mathematical model for positioning error is derived for single and multiple LED based IPS for circular, rectangular and square beam shapes. In addition, with the help of exhaustive parameter analysis in terms of 90th percentile value found from the cumulative distribution frequency (CDF) and average error for the proposed algorithms, this paper provides a large set of values for system parameters like beam radius, LED separation distance and receiver separation distance for which the error in position estimation is within sub-metre accuracy. The major contributions of this research paper include:

- Proposing a novel mathematical model for calculating positioning error for an OWC-based IPS. This mathematical model is based on the concept of raw moments and helps in validating the results and inferences of the geometrical simulation. This leads to closed form expressions for standard deviation and average error for single and multiple LED IPS with different shapes which can be useful in other research domains as well. 
- The proposed algorithms are evaluated by emulating an indoor wireless communication channel in LOS configuration which helps in conforming the inferences drawn from the results of the geometrical simulation.

- An exhaustive combinational analysis is carried out to determine optimal system parameter values for designing an efficient IPS for different indoor environments and desired positioning accuracy.

- The OBRIP and TRIP algorithms are improved by proposing new concepts such as positioning using previous location, remedy for receiver blockage in the TRIP algorithm and room scaling.

The organisation of the paper is as follows: Section 2 presents the literature survey followed by the Section 3 which discusses the simulation setup for the OBRIP and TRIP algorithms. In Section 4, a LOS mathematical propagation model for the OWC channel is presented followed by Section 5, which presents the performance comparison of the proposed algorithms to the proximity method. Section 6 discusses the mathematical model for a single and multiple LED IPS for different beam shapes. In Section 7, effect of various parameters and conditions are analysed followed by Section 8 , which includes the guidelines for installation. Finally, in Section 9, the major conclusions from the paper are discussed

\section{Literature Survey}

Among the existing positioning methods, the triangulation method utilises the geometric properties of the triangle to determine the absolute position of the receiver. In this method, the position of the receiver is estimated by measuring the distance of the receiver from multiple LED transmitters via received signal strength (RSS) [6], time of arrival (TOA) or time difference of arrival (TDOA) techniques [28], or by measuring the angles relative to multiple LED transmitters using angle of arrival (AOA) technique [19]. Though this method has led to the development of various IPS which show a significant level of accuracy, their design involves various assumptions and are either too expensive or unsuitable for practical implementation $[19,21]$. For time-based positioning, extremely accurate time measurement is required as the distance between the receivers is relatively short in indoor applications [29]. In the RSS-based localisation, it is often assumed that the receiver is placed parallel to the transmitter, which results in incorrect position estimation when the receiver angle is tilted [6,12]. In addition, the transmitted power in RSS-based localisation is uncertain which further creates a challenge for distance estimation [29]. IPS with multiple receivers requires fixed orientation for which specific angles and heights are assumed [5], but in practice the receiver is often not restricted by a specific angle or height. In recent research [30], a positioning system based on angle difference of arrival (ADOA) technique is proposed which takes into account arbitrary tilting angles of the receiver and claims an accuracy of less than a half meter using image sensors or photodiode (PD) arrays. However, this system requires signals from at least three LED transmitters for position estimation and is computationally expensive. In addition, the accuracy of this system directly depends upon the size of the spatial step and the search area. The main challenge with angulation based positioning is the use of multiple PD receivers or image sensors which increases the hardware complexity [31]. In [32], localisation with the help of single transmitter and single tilted receiver achieves an accuracy of less than $4 \mathrm{~cm}$ based on the experimental results. However, the analysis is performed for a relatively small indoor environment where the receiver is required to be calibrated to a particular direction before the positioning.

Fingerprinting is another positioning method that compares real-time measurement data to the fingerprints of the pre-measured signal features at all points in the room to estimate the position of the receiver. Positioning using fingerprinting method is less complex as it does not rely on any specific hardware or theoretical model, thus eliminating the need for complex calculation [33]. However, this method requires redesigning of the fingerprints database when there is any change to the indoor environment which can prove to be expensive [34]. As compared to these methods, proximity method is the simplest and most inexpensive positioning method used for designing an OWC-based IPS [35]. This method directly employs the optical signal transmitted by the LED to infer the position of the 
receiver which makes the proximity-based IPS hardware-friendly and less complex [36]. It is also less dependent on environment changes. However, the accuracy of this method is poor as it depends on the factors such as size of the room and the LED coverage area [37]. To further increase the accuracy of the proximity method, additional devices are used. A constructive method is proposed in [38-40] which uses 6-axis sensors to reduce the positioning error from several meters to the range of $0.3 \mathrm{~m}$ to $0.6 \mathrm{~m}$ for the OWC-based positioning system. However, the accuracy claimed by these systems highly depends on the accuracy of angulation data obtained from the 6-axes sensor.

Analysis of the existing positioning methods further leads to the identification of the challenge to separate optical signals from two or more different light sources which are carrying different decoded positional information [41,42]. This situation happens when the light beam regions of LEDs of the OWC system, which are transmitting their location information in the form of modulated optical signal, overlap. In recent research [43], the multiple LED estimation model (MLEM) is proposed to establish an explicit coordination between LEDs and separate signals at the receiver. In MLEM, the positional information is encoded using pulse duration multiplexing (PDM) and the data is modulated using on-off keying (OOK) to a higher frequency. It reduces the interference caused by multiple LEDs transmitting at the same time and at the same wavelength by using low duty cycle protocol and packet based PDM. In order to deduce the location of the receiver, MLEM uses both the content and pattern of reception of data carrying packets from single or multiple LEDs. In a separate research [44], to overhaul the overlapping problem caused by multiple unsynchronized light sources over a shared light medium the entire spectrum is channelized into multiple evenly-spaced sub-carriers and possible collisions among light sources are mitigated by employing random channel hopping technique. Experimental performance analysis of the MLEM model has shown promising results in separating optical signals, and thus it is preferred for signal power separation over the other signal separation techniques. Considering that interference in the overlapping light regions can be moderated, OBRIP and TRIP algorithms are proposed in [26,27], respectively, which helps to address the shortcomings of the proximity-based IPS. Unlike the proximity method, these algorithms employ the centroid of all the received signals to locate the object instead of assigning the coordinates of the strongest received signal. Thus, the overlapping regions are used as an advantage to increase the accuracy in position estimation. The system setup for both the algorithms is discussed in the following section.

\section{System Setup}

To conduct simulation-based performance analysis of the OBRIP and TRIP algorithms, a room with dimensions $10 \mathrm{~m} \times 10 \mathrm{~m} \times 3 \mathrm{~m}$ is considered. A $3 \times 3$ grid array of nine equally spaced LEDs are assumed to be installed on the ceiling of the room as shown in Figure 1. An object whose position is to be estimated is placed in the room. To ensure stable error values and simulate all possible positions, 25,000 randomly generated and uniformly distributed positions of the object at a height of $1 \mathrm{~m}$ from the floor plane of the room are used to perform the error calculations for position estimation. The LED light beams form a geometrical shape (circular in this case) when seen from the top view of the room. The midpoints of these regions are the first set of reference points for position estimation. The second set of reference points is obtained from the centroid points of the overlapping light beam regions.

\subsection{Optimal Beam Radius Indoor Positioning Algorithm}

In the OBRIP algorithm, each LED in the LED array continuously transmits its location information inside a room in the form of a modulated optical signal. This signal is intercepted by an object with an optical receiver. Based on its position, if the object receives signal from only one LED, the coordinates of that LED are assigned as the reference position of the object. However, if the object lies in an overlapping region and receives multiple signals from two or more LEDs, the centroid of all the received coordinates is assigned as the reference position of the receiver. In case the object lies in the non-coverage region where it receives no signal, the centre of the room is assigned as the 
reference position of the object. Figure 2 illustrates the working of the OBRIP algorithm with the help of a flowchart.

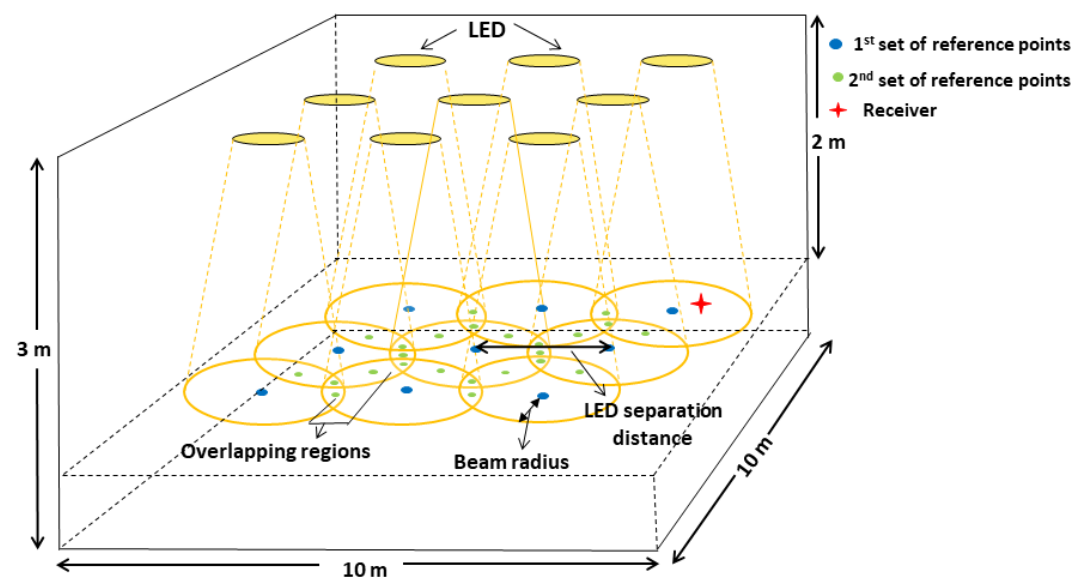

Figure 1. System setup for the OBRIP algorithm.

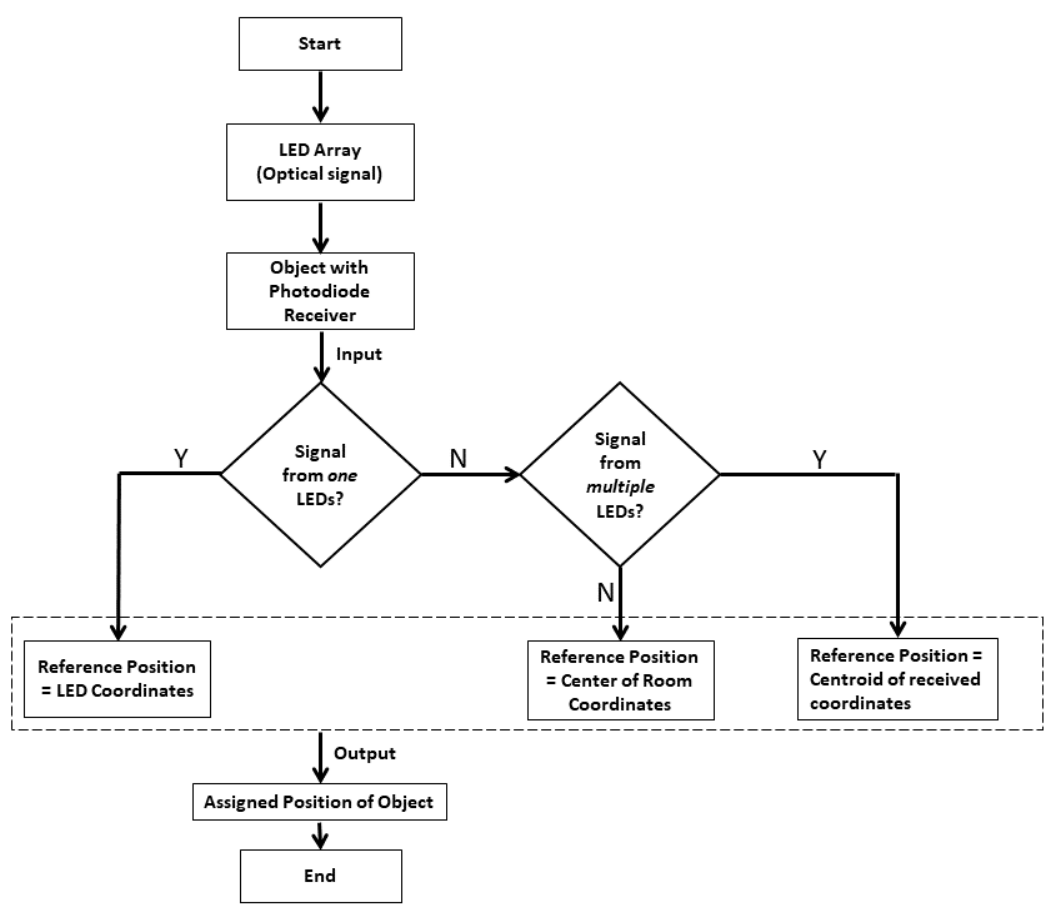

Figure 2. System flowchart for the OBRIP algorithm.

For the system setup discussed previously, consider a set of randomly generated positions of the receiver object, $\left(x_{A i}, y_{A i}\right), i=1, \cdots, N(N=25,000)$. For each of these random positions, an estimated position $\left(x_{R i}, y_{R i}\right)$ is determined using the OBRIP algorithm. Based on the randomly generated and estimated position of the receiver object in the $i$ th iteration, the average error in position estimation is calculated for the performance evaluation of the OBRIP algorithm. The mathematical expression for calculation of RMSE, or average error $(\bar{E})$, as referred to in this research, can be written as Equation (1):

$$
\bar{E}=\sqrt{\frac{1}{N} \sum_{i=1}^{N}\left(\left(x_{R i}-x_{A i}\right)^{2}+\left(y_{R i}-y_{A i}\right)^{2}\right)} .
$$




\subsection{Two-Receiver Indoor Positioning Algorithm}

The TRIP algorithm works with the same system setup as for the OBRIP algorithm with the exception that it employs two optical receivers on an object instead of one. When the receiver object enters a room, each of the receivers on the object independently receives one or more optical signals from the transmitting LEDs. The receivers then independently estimate their position using the principle of OBRIP algorithm. As per the TRIP algorithm, the final estimated position of the object is the midpoint of the estimated positions of both the receivers. Figure 3 shows the position assignment process in the TRIP algorithm with the help of a flow chart. For performance evaluation of TRIP algorithm, the average error in position estimation can be calculated using Equation (1).

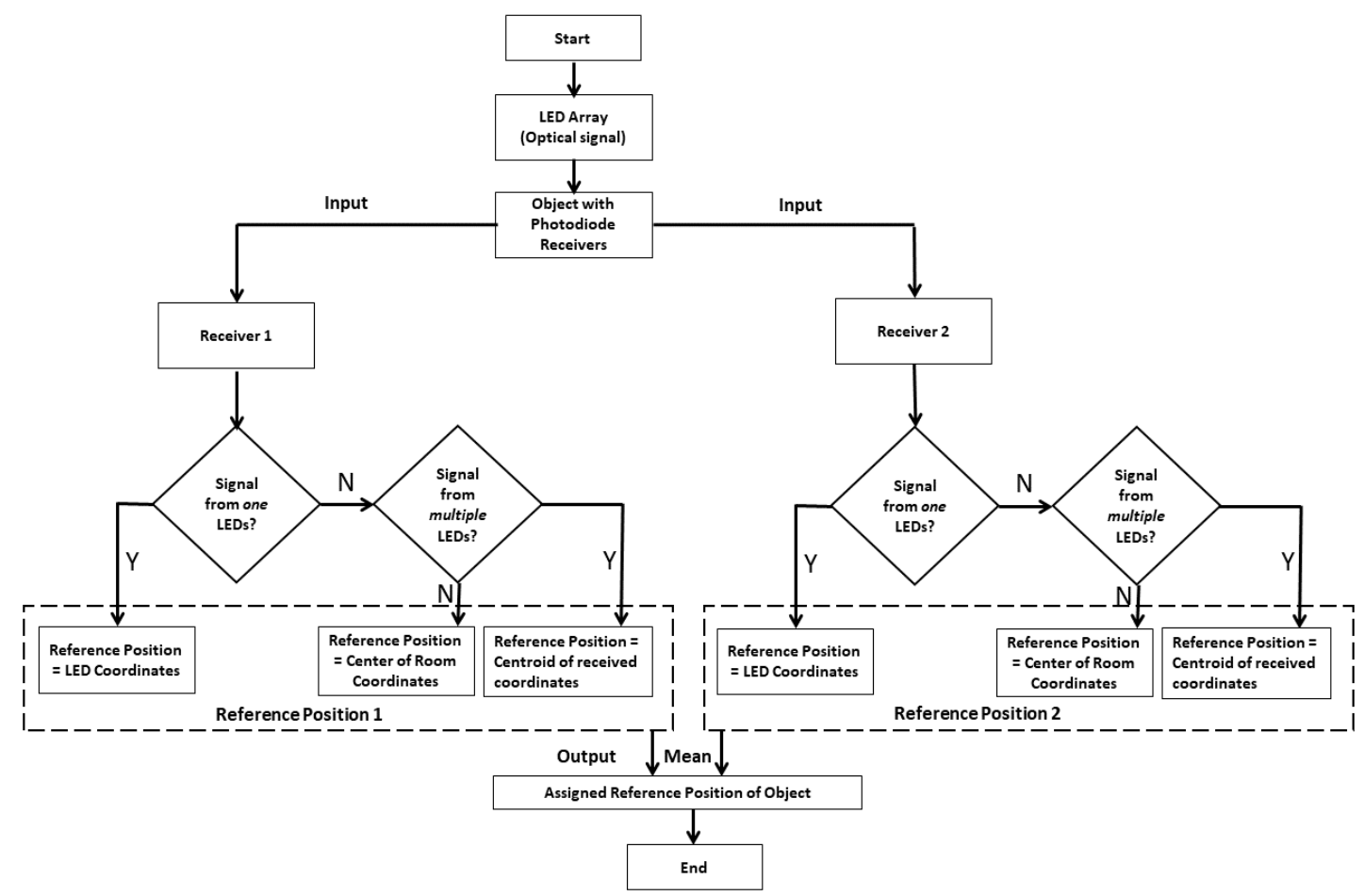

Figure 3. System flowchart for the TRIP algorithm.

In this research, the TRIP algorithm assumes the object to be an average size adult human being with an optical receiver attached to each of their shoulders. Therefore, the distance between the two receivers is kept $0.5 \mathrm{~m}$, which is the upper ceiling value of the average shoulder length for an adult human being [45]. In addition, as the algorithm is being designed for an IPS for elderly people with dementia, increasing the number of receivers beyond two can make the device uncomfortable, or impractical to wear. Furthermore, it will inherit the issue of angular dependency and the performance of the algorithm will have to be analysed as a three-dimensional problem.

\section{Line of Sight Propagation Model for an Optical Wireless Communication Channel}

Understanding the characteristics of the underlying communication channel associated with an indoor environment is imperative to designing an efficient indoor optical wireless positioning system. The impulse response of the channel is used to characterize the OWC channel. Considerable research has been done to model this communication channel and various experimental and computer-generated models have been proposed [46].

In an indoor optical wireless channel, the link length is relatively short because of which scattering and absorption due to attenuation is negligible [46]. Considering a simulation setup similar to OBRIP and TRIP algorithms as described in the previous section, along with an OWC link with a Lambertian 
source, a PD receiver, an optical band-pass filter of transmission $T_{S}(\psi)$ and a nonimaging concentrator of gain $g(\psi)$, a LOS propagation model, as shown in Figure 4, is designed to approximate the DC channel gain, $H(0)$, for individual LED transmitters. LOS propagation channel takes into consideration the optical signals which travel in a direct path to the receiver without any obstruction. Equations (2)-(5) are derived to model a LOS optical wireless channel [28,46-50]:

$$
H(0)= \begin{cases}\frac{A_{r}(m+1)}{2 \pi d_{t r}^{2}} \cos ^{m}(\phi) g(\psi) T_{S}(\psi) \cos (\psi), & 0 \leq \psi \leq \psi_{c} \\ 0, & \text { otherwise }\end{cases}
$$

where $A_{r}$ is the area of the receiver, $d_{t r}$ is the LOS transmitter-receiver separation, $\phi$ is the angle of irradiance and $\psi$ is angle of incidence as shown in Figure 4. $T_{\mathcal{S}}(\psi)$ is the gain of the optical filter, $g(\psi)$ is the gain of the optical concentrator and $\psi_{c}$ denotes the field of vision (FOV) of the receiver. The order of Lambertian emission, $m$, can be expressed as function of LED semi-angle at half power, $\Phi_{1 / 2}$ :

$$
m=\frac{-\ln 2}{\ln \cos \left(\Phi_{\frac{1}{2}}\right)} .
$$

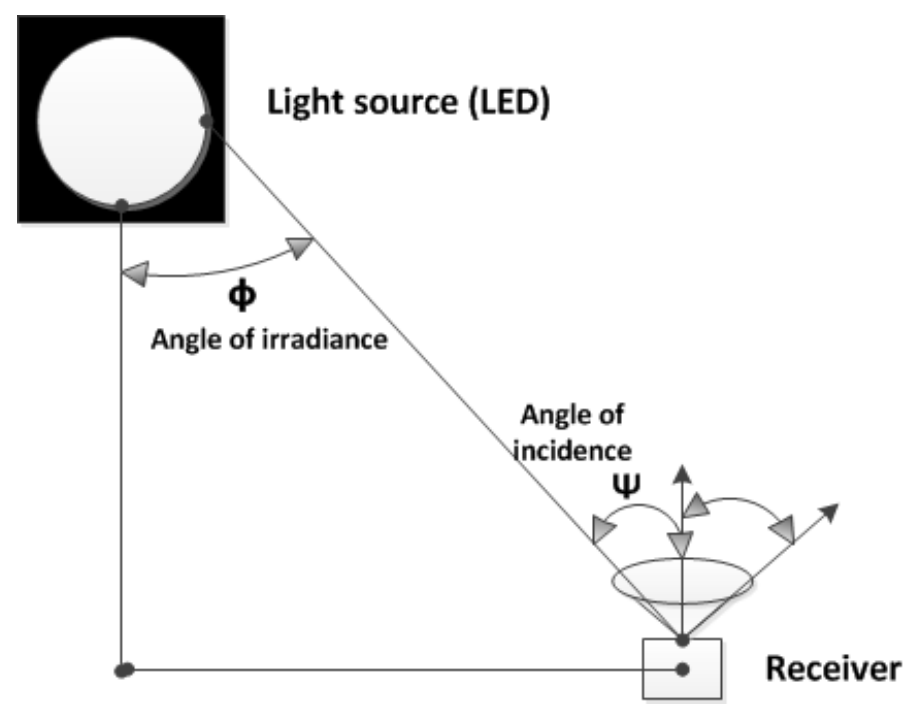

Figure 4. Line of sight model.

The gain of the concentrator $g(\psi)$ depends upon the internal refractive index of the concentrator $n$ and is given by

$$
\mathrm{g}(\psi)= \begin{cases}\frac{n^{2}}{\sin ^{2}\left(\psi_{c}\right)}, & \text { for } 0 \leq \psi \leq \psi_{c} \\ 0, & 0 \geq \psi_{c}\end{cases}
$$

The power received by the receiver inside a room depends on the optical power transmitted by individual LEDs. This received power, $P_{r}$, can be written as

$$
P_{r}=P_{t} H(0),
$$

where $P_{t}$ is the optical power transmitted by individual LEDs. Table 1 shows the parameter values which have been considered for the calculation of optical power received by the receiver and correspondingly the error in position estimation. It is initially assumed that $\phi$ and $\psi$ are equal to each other i.e., the surface of the receiver is placed parallel to the transmitter and the surface of the room. For the purpose of performance analysis, tilting of the receiver surface is also considered. 
Table 1. Parameters used for simulation.

\begin{tabular}{cc}
\hline Semi-angle at half power $\Phi_{\frac{1}{2}}$ & $30^{\circ}$ \\
Gain of the optical filter $T_{S}(\psi)$ & 1 \\
Refractive index $n$ & 1.5 \\
Field of view $\psi_{c}$ & $80^{\circ}$ \\
Optical power transmitted by each LED $P_{t}$ & $20 \mathrm{~mW}$ \\
Physical area of detector in a PD $A_{r}$ & $1.0 \mathrm{~cm}^{2}$ \\
\hline
\end{tabular}

\section{Performance Comparison of Proposed Algorithms with Proximity Method}

The proposed algorithms claim to improve the performance of the proximity method by taking the advantage of overlapping regions to reduce the error in position estimation. In this research, the performance comparison between the proximity method, OBRIP and TRIP algorithms are carried out using the system setup discussed above.

For the purpose of analysis, the average error is estimated for the three methods with change in beam radius. The simulation results shown in Figure 5 are for beam radius values ranging from $1.5 \mathrm{~m}$ to $3.5 \mathrm{~m}$ where the effect of overlapping regions is noticeable. It can be observed that the average error difference between the OBRIP and the proximity method keeps on increasing as the area of the overlapping regions increases with beam radius. After a certain point, when the area of the overlapping region becomes larger than the individual LED coverage area, thus increasing positional uncertainty, the difference in performance of the two techniques starts reducing. At the optimal point, where the error is minimum in the case of OBRIP algorithm, OBRIP improves the accuracy of the proximity method by $28 \%$. The TRIP algorithm further reduces the error obtained from the OBRIP algorithm by up to $30 \%$. This analysis proves that the proposed algorithms lead to a superior performance as compared to the proximity method.

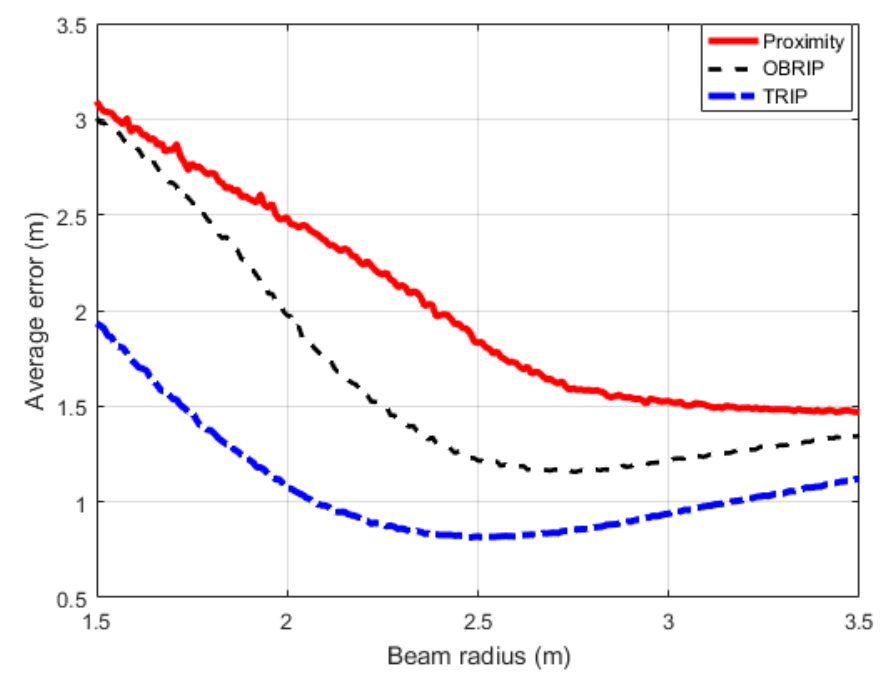

Figure 5. Average error vs. beam radius for proximity method, OBRIP algorithm and TRIP algorithm.

\section{Mathematical Model for Indoor Positioning System}

The concept of raw moments is used to derive a mathematical model to find the standard deviation of randomly distributed points around the centre of a geometrical shape [51]. Raw moment $\mu_{\mathrm{n}}$, or 'crude moment', is the $n$-th moment about zero of a probability density function $f(x)$ [52].

In this paper, the mean represents the position coordinates of the LED, or reference point for position estimation. The second raw moment is used for the calculation of standard deviation of uniformly distributed random position around the mean within a finite radius, which corresponds to 
beam radius of the LED. With the value of mean being 0 , the second moment about the mean $\mu_{2}$ is equal to the variance. Standard deviation, $\sigma$, can be expressed as the square root of variance [51]:

$$
\sigma=\sqrt{\mu_{2}}
$$

In Section 6.1, a brief study is presented to discuss the closed form expressions used to find the standard deviation for a single LED IPS having circular, rectangular and square beam shapes.

\subsection{Single LED Indoor Positioning System}

There exists a large amount of research to find the standard deviation of uniformly distributed random points around the centre of a circle, rectangle or a square [53-56], which gives closed form expressions for the second raw moment of these geometrical shapes. In this paper, these closed form expressions are used to find the standard deviation for different values of beam radius using the normalised second raw moment with respect to area of the shape, and then compared with simulation results.

For a circular beam shape, there exists an expression for standard deviation in terms of radius $r$. This represents the average error in position estimation in case of an LED with a circular beam shape and beam radius $r$. For the purpose of calculation, the LED position coordinates are taken as $(0,0)$. The standard deviation of uniformly distributed random points around the centre of a circle with radius $r$ can then be written as [53,57]:

$$
\sigma_{c}=\frac{r}{\sqrt{2}}
$$

As the number of LEDs with circular beam shape used for indoor positioning increases, it also becomes increasingly difficult to mathematically validate the results of simulation for the proposed algorithms. This is because overlapping of the light beam regions of these LEDs gives rise to new shapes for which standard deviation has to be calculated. However, if a new quadrilateral beam shape, like a square or rectangle, is designed, keeping half the length of the shorter side equal to the beam radius of the circular beam shape, any number of overlapping regions will always lead to creation of a new quadrilateral shape for which standard deviation calculation is easier. For the purpose of analysis, exhaustive simulation is carried out to find the average error in position estimation, as discussed in the following section (Section 7), and it is observed that, for rectangular and square beams shapes, average error is similar to that obtained for a circular beam shape. In addition, according to an energy mapping technique proposed in a recent research, it is possible to obtain LED beam patterns [58] with varying geometrical shapes, like square or rectangle, and uniform distribution of light intensity. Taking these points into consideration, if a mathematical expression exists for the standard deviation of a rectangle, it will also help in validating the mathematical model for a larger system of LEDs with varying beam shapes.

For a rectangular beam shape with length $h$ and breadth $b$, standard deviation $\sigma_{r}$ can be expressed as [54-57]:

$$
\sigma_{r}=\sqrt{\frac{h^{2}}{12}+\frac{b^{2}}{12}}
$$

Considering that square is a special case of a rectangle where the length and breadth are equal, Equation (9) is used to derive the expression for standard deviation for a square beam shape with side $s$ :

$$
\sigma_{s q}=\sqrt{\frac{s^{2}}{6}} .
$$

As the focus of this paper is on overlapping light beam regions, the expressions presented in this section are used to derive the closed form expressions for standard deviation for a multiple LED IPS, as discussed in Section 6.2. 


\subsection{Multiple LED Indoor Positioning System}

As discussed in the previous section, there is ample existing research to find the standard deviation of uniformly distributed points around the centre of a circle or a rectangle, but, surprisingly, no closed form expression exists for the standard deviation of two overlapping circles. One of the main inferences of the OBRIP algorithm is that increase in the number of overlapping regions leads to reduction in average error in position estimation [26,27]. In this paper, an expression for standard deviation is derived by taking two overlapping circles, with beam radius $r$ and distance between centres as $2 d$, where $d$ is the distance between the centre of the circle and eye, while validating the inferences of the proposed positioning algorithm.

As can be seen in Figure 6, overlapping circles lead to formation of three regions, Region 1 and Region 3 which are in the shape of a 'crescent' and are identical, and Region 2, which is in the shape of an 'eye'. As per the OBRIP algorithm each region has its own reference point. According to the concept of raw moments, the expression for the second moment of the eye shape $\mu_{2 e}$ can be written as:

$$
\mu_{2 e}=\int_{0}^{\frac{\pi}{2}} \int_{0}^{U_{l}} r^{2}(r \mathrm{~d} r \mathrm{~d} \theta),
$$

where

$$
U_{l}=-d \cos \theta+\sqrt{d^{2}\left(\cos ^{2} \theta-1\right)+r^{2}}, \quad \theta=\cos ^{-1} \frac{d}{r} .
$$

The solution for (10) is given in Appendix A, which leads to the expression for the second moment of the eye shape around its centre, $\mu_{2 e}$ :

$$
\mu_{2 e}=d^{4}\left(c(c+2) \tan ^{-1}(\sqrt{c-1})-3 c \sqrt{c-1}\right),
$$

where

$$
c=\frac{r^{2}}{d^{2}} .
$$

The standard deviation of the eye shape, $\sigma_{e}$, can be obtained from the normalised second raw moment of the eye shape:

$$
\sigma_{e}=\sqrt{\frac{\mu_{2 e}}{A_{e}}}
$$

where $A_{e}$ is given as:

$$
A_{e}=r^{2}\left(2 \cos ^{-1} \frac{d}{r}-\sin \left(2 \cos ^{-1} \frac{d}{r}\right)\right) .
$$

After calculating the standard deviation for the eye shape, the next task is to find the standard deviation for the crescent shapes, $\mu_{2 c r}$. This value can be calculated if second moment for the eye shape around the centre of circle, $\mu_{2 e c}$, is subtracted from the second moment of an entire circle, $\mu_{2 c}$,

$$
\mu_{2 c r}=\mu_{2 c}-\mu_{2 e c},
$$

where

$$
\mu_{2 c}=\frac{\pi}{2} r^{4} .
$$

$\mu_{2 e c}$ can be written as a function of second moment of the eye around its own center, $\mu_{2 e}$, area of the eye, $A_{e}$, and $d$ :

$$
\mu_{2 e c}=\mu_{2 e}+A_{e} d^{2} .
$$

The standard deviation of the crescent shape can now be written as a function of the second moment of the crescent shape and area of crescent shape as shown in Equation (19): 


$$
\sigma_{2 c r}=\sqrt{\frac{\mu_{2 c}-\mu_{2 e c}}{A_{c}-A_{e}}}
$$

where

$$
A_{c}=\pi r^{2} .
$$

To analyse the effect of change in overlapping region area on average error, two LEDs with circular beam shapes and a fixed beam radius of $3 \mathrm{~m}$ are brought close to each other, and change in average error in position estimation is observed. To find the overall error of the entire system with the eye and crescent shapes, the weighted average method is used as given in (21):

$$
\sigma_{2 c}=\left(\frac{2 \sigma_{2 c r} A_{c r}+\sigma_{e} A_{e}}{2 A_{c r}+A_{e}}\right),
$$

where

$$
A_{c r}=A_{c}-A_{e} .
$$

The overlapping distance, $2(r-d)$, is then varied and the average error of the entire system is found using the mathematical model and geometrical simulation, and plotted in Figure 7 . The initial observation is that average error decreases rapidly with increase in overlapping distance up to a certain minimum point. This decrease can be attributed to the increase in the area of the overlapping regions which encompasses more number of random points for position estimation. After this minimum point when the overlapping distance is further increased, the area of the overlapping region becomes larger than the individual LED beam area. This results in increasing the positional uncertainty, thus the error starts increasing again. The results obtained from both the mathematical model and geometrical simulation are identical which validates the accuracy of the approach.

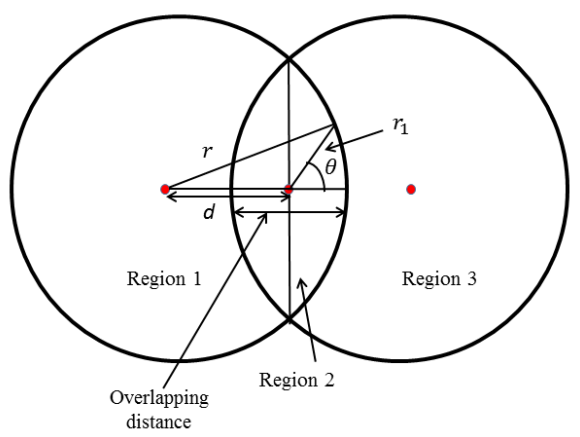

Figure 6. Two overlapping circular LED beams.

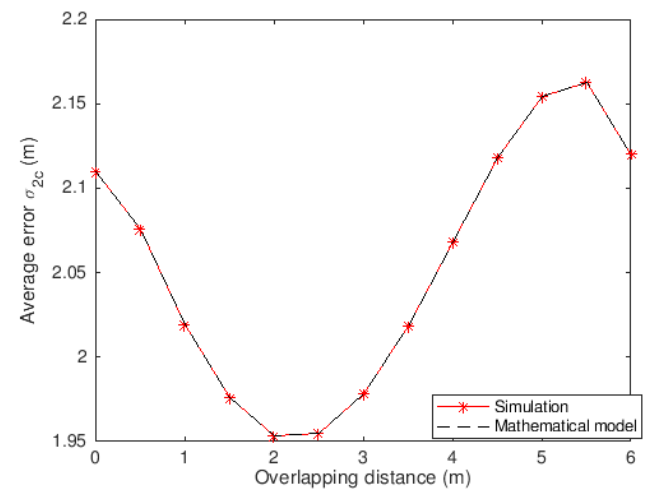

Figure 7. Average error $\left(\sigma_{2 c}\right)$ vs. overlapping distance for two overlapping LED beams (circular beam shape). 
The two LED-system with room edge effects can then be considered as three different rectangles as shown in Figure 8. The edge effect occurs when the LED light beam regions are cut-off by the opaque walls of the room. Using Equation (8), standard deviation for each of these rectangles can be calculated. The overall standard deviation of the system is then calculated using the weighted average expression which can be written as:

$$
\sigma_{2 r}=\left(\frac{2 E_{1} \mathrm{~A}_{1}+E_{2} A_{2}}{2 A_{1}+A_{2}}\right),
$$

where $E_{1}$ and $A_{1}$ are the standard deviation and area of the two similar beam regions (Region 1 and Region 3), respectively, and $E_{2}$ and $A_{2}$ represent the standard deviation and area of the middle light beam region (Region 2), respectively. The equations used to find the values of these variables are given in Appendix B. For a system with more LEDs, a similar approach can be used to find the expression for overall standard deviation.

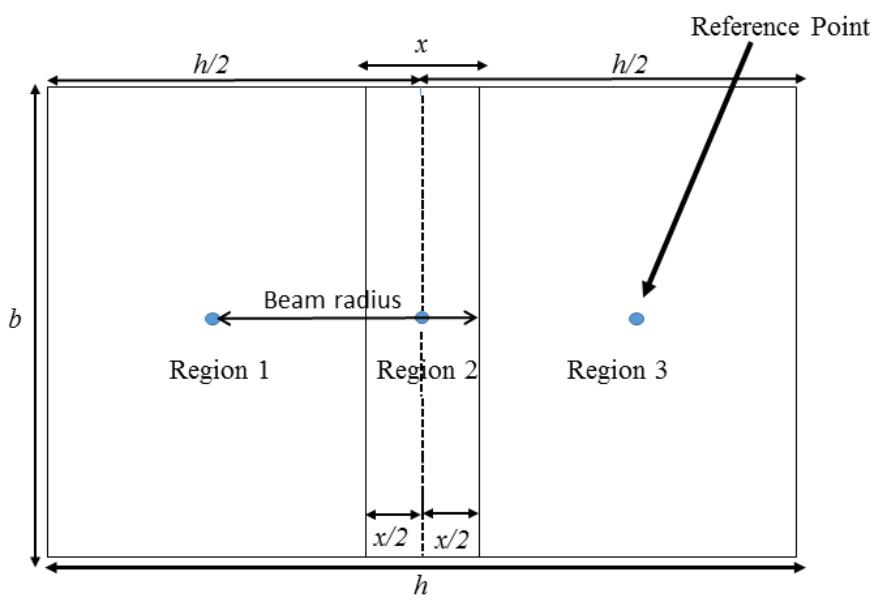

Figure 8. Two overlapping rectangular LED beams.

Using (23), the overall average error of the three-rectangle system is plotted against beam radius (half the length of the shorter side of the rectangle) as shown in Figure 9. It can be observed that, as the overlapping of the two LEDs increases, the average error starts decreasing, but after a certain 'optimal' point, when the area of the overlapping region becomes larger than the area of actual LED light beam, error in position estimation starts increasing again. This optimal point occurs at a beam radius of $4.17 \mathrm{~m}$ when the standard deviation is $3.04 \mathrm{~m}$ (Case I).

In Figure 8, the condition with two rectangular overlapping light beams in a room with length $h$ and breadth $b$ is shown. Taking the length of the overlap region as $x$, this paper also tries to find the relationship between $x$ and the actual length of the room $h$, where the average error is minimum, by using the method of derivatives. Going by intuition, the minimum standard deviation should occur when the area of all the regions is same, which leads to the occurrence of three equal rectangular regions i.e., the length of each of the region is $h / 3$. To confirm this, the method of derivatives is applied on (23), which also gives the relation, $x=h / 3$, at which minimum error occurs. The calculation to get this expression is shown in Appendix B. The value of standard deviation at this condition can be written as:

$$
\sigma_{2 r m}=\sqrt{\frac{\frac{h^{2}}{9}+b^{2}}{12}} .
$$

Using the above derived relationship, for a room of size $10 \mathrm{~m} \times 10 \mathrm{~m}$, minimum standard deviation should occur when the length of each region is $3.33 \mathrm{~m}$, or all regions have the same area, $3.33 \mathrm{~m} \times 10 \mathrm{~m}$. This situation is possible when the length of the actual LED light beam with the room edge effect is $8.33 \mathrm{~m}$ or the beam radius is $4.17 \mathrm{~m}$. This is the same value as obtained from the plot 
between beam radius and average error from the simulation results as shown in Figure 9 (Case I). In addition, using (24), the minimum standard deviation value obtained is $3.04 \mathrm{~m}$, which is same as the value obtained from the simulation.

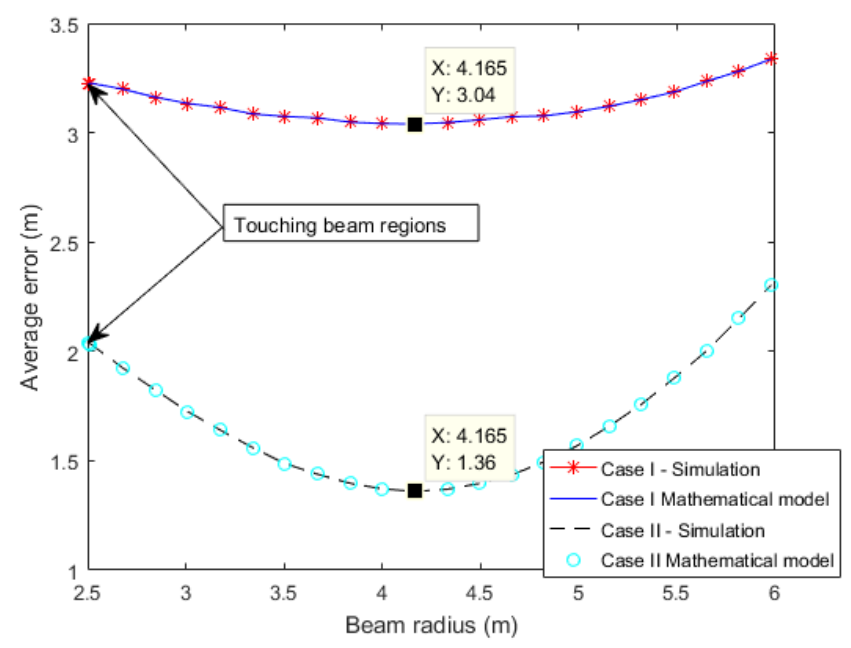

Figure 9. Average error vs. beam radius for two (Case I) and four (Case II) overlapping rectangular LED beams (simulation and mathematical model).

Based on the intuition and the results obtained from two rectangular LED beams, a generalised scenario is considered where an array of $m \times n$ LEDs is installed in a room with length $h_{g}$ and breadth $b_{g}$. This results in $2 m-1 \times 2 n-1$ light beam regions. As discussed previously, minimum error should occur when all the resulting light beam regions have equal areas. Under this condition, the overlapping distance along the length, $x_{g}$, and along the breadth, $y_{g}$, of the room can be written as a function of $h_{g}$ and $n$, and $b_{g}$ and $m$ as given by (25) and (26), respectively:

$$
\begin{aligned}
& x_{g}=\frac{h_{g}}{2 n-1}, \\
& y_{g}=\frac{b_{g}}{2 m-1} .
\end{aligned}
$$

The approximate value of minimum standard deviation for this system is given by (27) and can be used to calculate standard deviation for a room with any size and any number of LED transmitters:

$$
\sigma_{g}=\sqrt{\frac{\left(\frac{h_{g}}{2 n-1}\right)^{2}+\left(\frac{b_{g}}{2 m-1}\right)^{2}}{12}} .
$$

The value of beam radius for this system, $r_{g}$, can be expressed as:

$$
r_{g}= \begin{cases}\frac{(3 n-1) h_{g}}{2 n(2 n-1)}, & \frac{h_{g}}{n} \leq \frac{b_{g}}{m}, \\ \frac{(3 m-1) b_{g}}{2 m(2 m-1)}, & \frac{h_{g}}{n} \geq \frac{b_{g}}{m} .\end{cases}
$$

For $m=n$ and $h_{g}=b_{g}$, the approximate value of minimum standard deviation is:

$$
\sigma_{g}=\frac{h_{g}}{(2 n-1) \sqrt{6}} .
$$

One of the main conclusions from the simulation of the proposed algorithm is that, with an increasing number of LEDs, the overall error reduces due to an increase in the number 
of overlapping regions. This is because more overlapping regions lead to a larger number of reference points which reduces the distance between the actual and estimated position of the object. Using (29), the minimum standard deviation for four $(2 \times 2)$ and nine $(3 \times 3)$ LED systems in a room with dimensions $10 \mathrm{~m} \times 10 \mathrm{~m}$ comes out to $1.36 \mathrm{~m}$ and $0.82 \mathrm{~m}$, respectively. This result matches the result obtained from simulation for four overlapping rectangular LED systems exactly, as shown in Figure 9 (Case II). For nine overlapping rectangular beams, this value is very close to the simulation results as shown in Figure 10, but not an exact match. This is because, in the case of nine overlapping rectangular beams, there does not exist a situation where all overlapping region areas are same. Thus, the generalised equation derived above gives the exact error value for a smaller number of rectangular LEDs, but, as the number of LEDs increase, the error value does not exactly match, but is very close to simulation results. In addition, when these error values are compared to a two LED systems for the same room size (Case I), the error values for a four and nine LED system are considerably less, as can also be observed in Figures 9 and 10. This validates the inference of the proposed algorithm that increasing the number of overlapping regions causes further reduction in average error values.

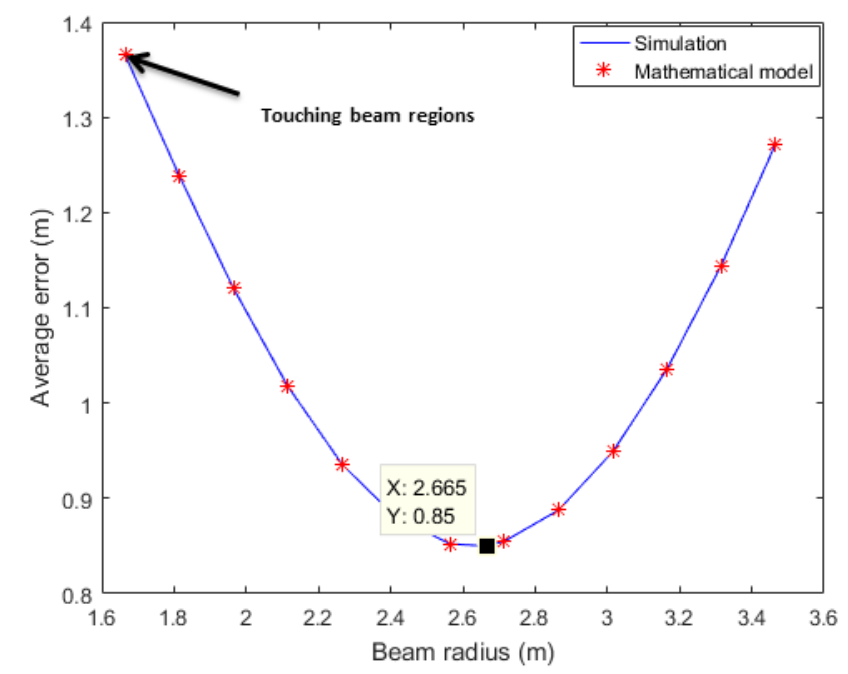

Figure 10. Average error vs. beam radius for nine overlapping rectangular LED beams (simulation and mathematical model).

For LEDs with circular beam shapes, in case of more than two overlapping beams, calculating the error in position estimation can become more complex. However, looking closely at Figure 11, it can be observed that the multiple LED system can be viewed as a combination of various shapes like rectangles and triangles. Triangles, in this case, can be considered as one-fourth the area of a rectangle, and therefore equations stated previously can be used to find the standard deviation of these shapes and consequently the entire system. In case of an LED system with a numerous positioning of LEDs in a very large room, it can be thought of as a repeating smaller grid of LEDs. Thus, if the effect of the edges of the room is excluded, the error can be calculated for the smaller grid and used to generalise for the entire system. 


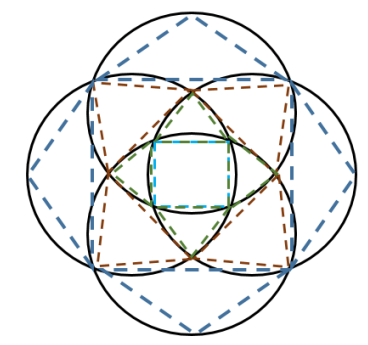

Figure 11. Multiple overlapping LED light beams.

\section{Performance Analysis of OBRIP and TRIP Algorithm}

\subsection{OBRIP and TRIP Algorithms with Channel Modelling}

In the related research, the performance analysis of OBRIP and TRIP algorithms is carried out without considering the effect of channel parameters. It is observed that TRIP outperforms OBRIP and leads to further reduction in average error in position estimation by $30 \%$ which is also exhibited in Figure 12. To validate these findings in the presence of an optical wireless channel, an LOS propagation model, as discussed in Section 4, is simulated and the performance of OBRIP and TRIP algorithms are analysed. On comparison, the results of the non-channel implementation of OBRIP and TRIP algorithms are identical to the implementation incorporating the indoor optical wireless channel as shown in Figure 12. This also confirms that further reduction of 30\% in average error is possible using TRIP algorithm as compared to OBRIP algorithm.

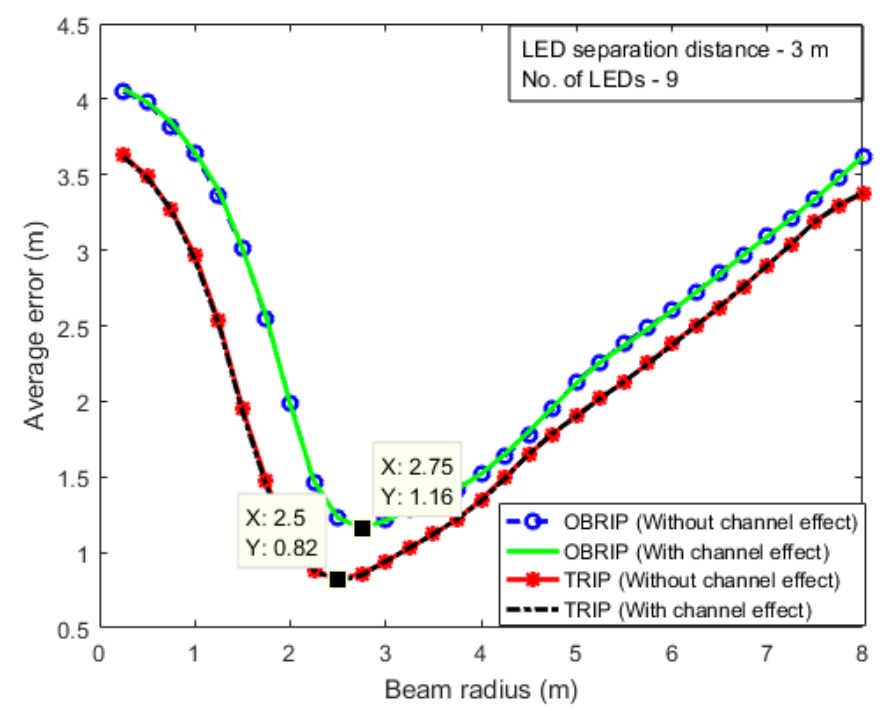

Figure 12. OBRIP and TRIP algorithms with and without channel modelling.

\subsection{Optimisation of System Parameters}

In order to find the optimal values for the system parameters, beam radius and LED separation distance, an exhaustive simulation is performed with various possible combinations of values for both of these parameters in the presence of an optical wireless channel. The beam radius is varied from $0.25 \mathrm{~m}$ to $8 \mathrm{~m}$ at intervals of $0.05 \mathrm{~m}$, and for each value of the beam radius, the LED separation distance is varied from $1.5 \mathrm{~m}$ to $5 \mathrm{~m}$ at intervals of $0.25 \mathrm{~m}$. For each of these 2340 combinations of beam radius and LED separation distance, the error in position estimation is then calculated. These error values are used to find the 90th percentile error and the average error in position estimation. The 90th percentile error value is found from the CDF plot of the error values for each of the combinations of beam radius and LED separation distance. In order to get the complete picture for designing an IPS, 
both performance metrics are required. These metrics can help in the selection of a range of values for beam radius and LED separation distance to adapt any room size and shape, while providing acceptable values of positioning error.

In Figure 13a,b shown for selected values of LED separation and beam radius, it can be observed that, for the channel-included OBRIP algorithm simulation, the smallest 90th percentile error obtained is $1.2 \mathrm{~m}$ at a beam radius of $3 \mathrm{~m}$ and LED separation distance of $3.75 \mathrm{~m}$. From the results of the simulation, in terms of average error, the smallest average error of $0.81 \mathrm{~m}$ is obtained at a beam radius of $3.4 \mathrm{~m}$ and separation of $4 \mathrm{~m}$. For the TRIP algorithm, the smallest 90th percentile error value is $0.94 \mathrm{~m}$ at a beam radius of $3 \mathrm{~m}$ and LED separation distance of $3.75 \mathrm{~m}$. In terms of average error, the smallest average error obtained is $0.61 \mathrm{~m}$ at a beam radius of $3.2 \mathrm{~m}$ and a separation of $3.75 \mathrm{~m}$. These exhaustive simulation results confirm the practical feasibility of proposed indoor positioning algorithms as error values are within sub-metre accuracy for various combinations of system parameters.

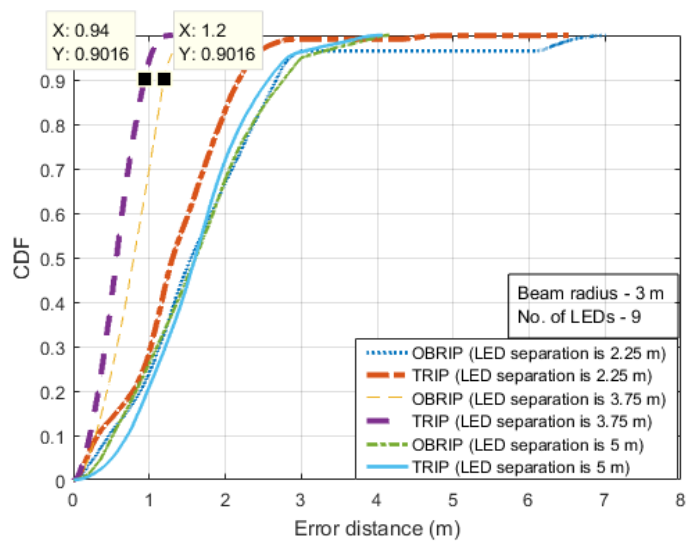

(a)

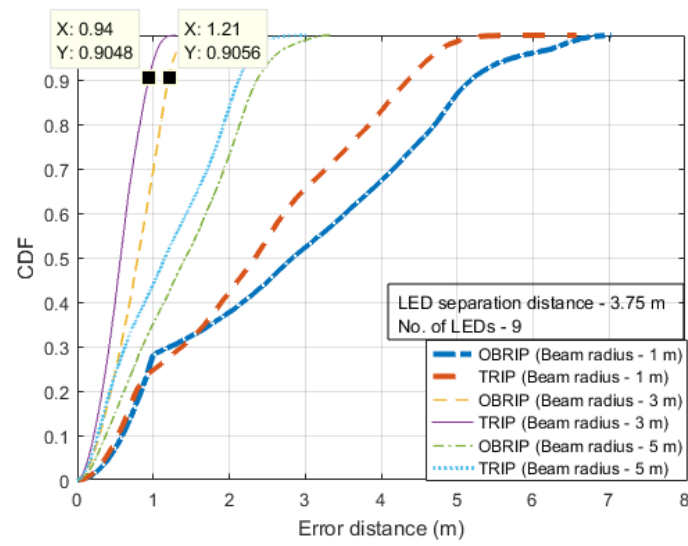

(b)

Figure 13. CDF distribution for OBRIP and TRIP algorithms (a) different LED separation distance; (b) different beam radius of LEDs.

The performance of TRIP and OBRIP can also be compared through the contour plots shown in Figure 14a,b obtained from the simulation performed above. It can be observed that, for the TRIP algorithm, the $1 \mathrm{~m}$ average error contour line covers a larger area and thus a larger set of values of beam radius and LED separation distance lead to error values of $1 \mathrm{~m}$ or less as compared to OBRIP, which demonstrates that the TRIP algorithm's performance is more robust to variability of system parameters than the OBRIP algorithm.

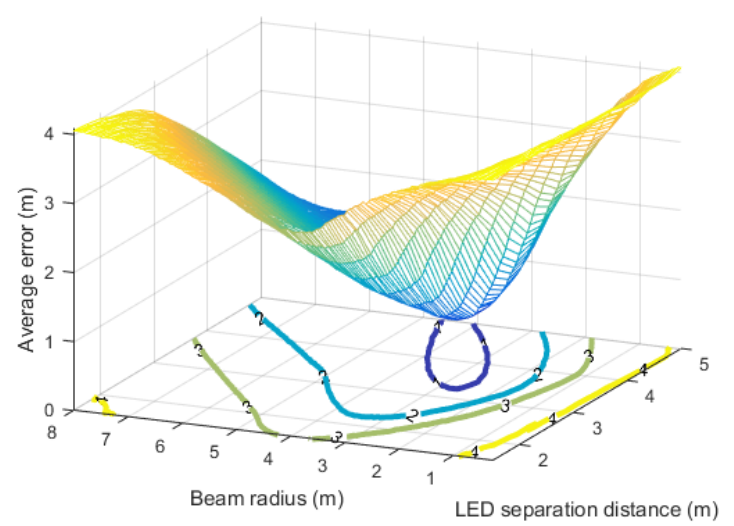

(a)

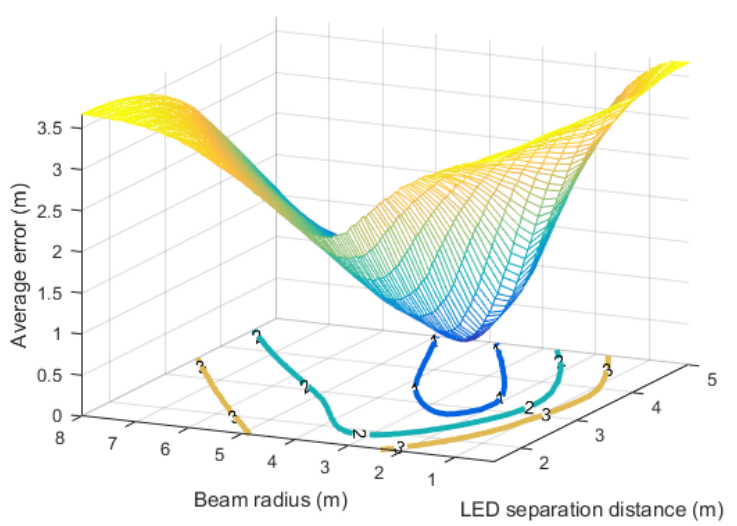

(b)

Figure 14. Average error vs. beam radius vs. LED separation distance (a) OBRIP; (b) TRIP. 


\subsection{Receiver Tilting}

Up until now, it is assumed that the receiver surface is always parallel to the LED array plane. To prove the robustness of the OBRIP and TRIP algorithms, the receiver angle is varied and corresponding average error is calculated. All randomly generated positions of the receiver are tilted by the same angle, from $-25^{\circ}$ to $25^{\circ}$ at intervals of $5^{\circ}$. From the detailed analysis, it is observed that the minimum average error of $1.16 \mathrm{~m}$ and $0.82 \mathrm{~m}$ is obtained at a beam radius of $2.75 \mathrm{~m}$ and $2.5 \mathrm{~m}$, when the receiver tilting angle is $0^{\circ}$ (i.e., no tilting) for OBRIP and TRIP algorithms, respectively. On comparing the contour plots shown in Figure 15a,b, where the contour lines represent the average error in position estimation, it is observed that, for TRIP, a larger set of beam radius and receiver tilting angles combinations produce an average error of $1 \mathrm{~m}$ or less as compared to OBRIP.

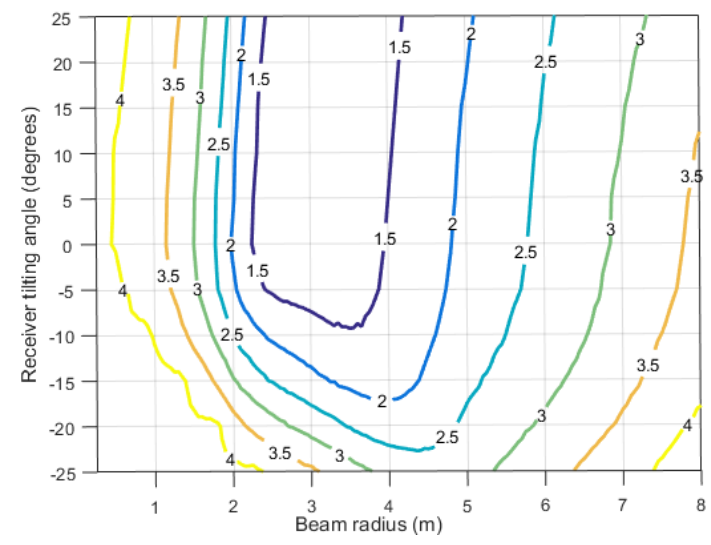

(a)

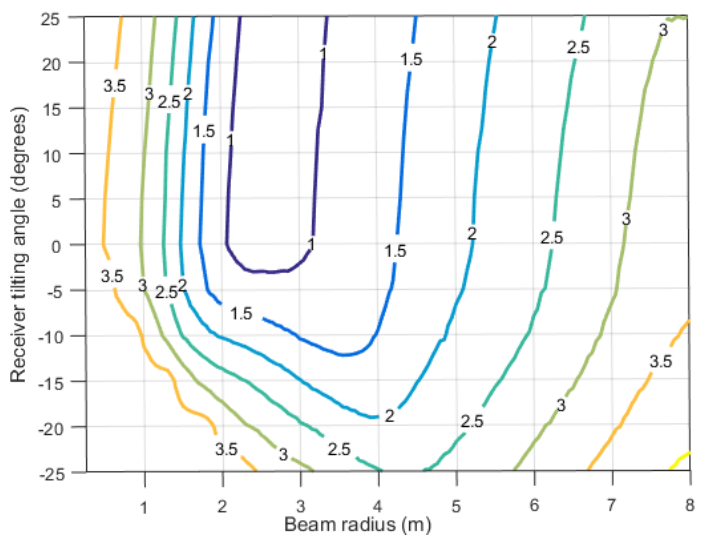

(b)

Figure 15. Implementation of proposed algorithms with different receiver tilting angles (a) OBRIP;

(b) TRIP.

\subsection{Distance between Two Receivers in a TRIP Algorithm}

In the performance analysis of TRIP algorithm, the distance between the two receivers is kept constant at $0.5 \mathrm{~m}$ until now. This leaves the window of opportunity to analyse the effect of varying this separation on average error in position estimation. In this section, the distance between the receivers is varied in the presence of channel parameters from $0.25 \mathrm{~m}$ to $5 \mathrm{~m}$, in steps of $0.25 \mathrm{~m}$. This range of receiver distances should be able to include everything from the shoulder length of an average adult human being to large shipment containers in warehouses. From the simulation results of selected receiver separations shown in Figure 16, it is observed that the minimum average error is obtained at a beam radius of $2.5 \mathrm{~m}$. In addition, as the receiver distance increases, up to $3.5 \mathrm{~m}$, the average error values keep on decreasing, after which no significant variation in average error is noticeable at higher values of beam radius.

A detailed analysis is also performed using the values of beam radius and LED separation distance obtained from analysis performed in Section 7.2, which gives minimum average error to observe the effect on average error when the distance between two receivers is varied from $0.25 \mathrm{~m}$ to $5 \mathrm{~m}$. Two cases are considered to observe this effect. In the first case, separation between the LEDs is fixed at $3.75 \mathrm{~m}$ and beam radius is varied from $2.5 \mathrm{~m}$ to $3.5 \mathrm{~m}$ in steps of $0.20 \mathrm{~m}$. In the second case, the beam radius is fixed at the value of $3.2 \mathrm{~m}$ and the separation between the LEDs is varied from $3 \mathrm{~m}$ to $4.25 \mathrm{~m}$ in steps of $0.25 \mathrm{~m}$. From the simulation results for the two cases above shown for some values in Figure 17a,b, respectively, it is observed that the average error values are under $1 \mathrm{~m}$ for majority of receiver separation distances. Thus, TRIP is giving a large range of operability values for LED separation distance, beam radius and receiver separation distance. 


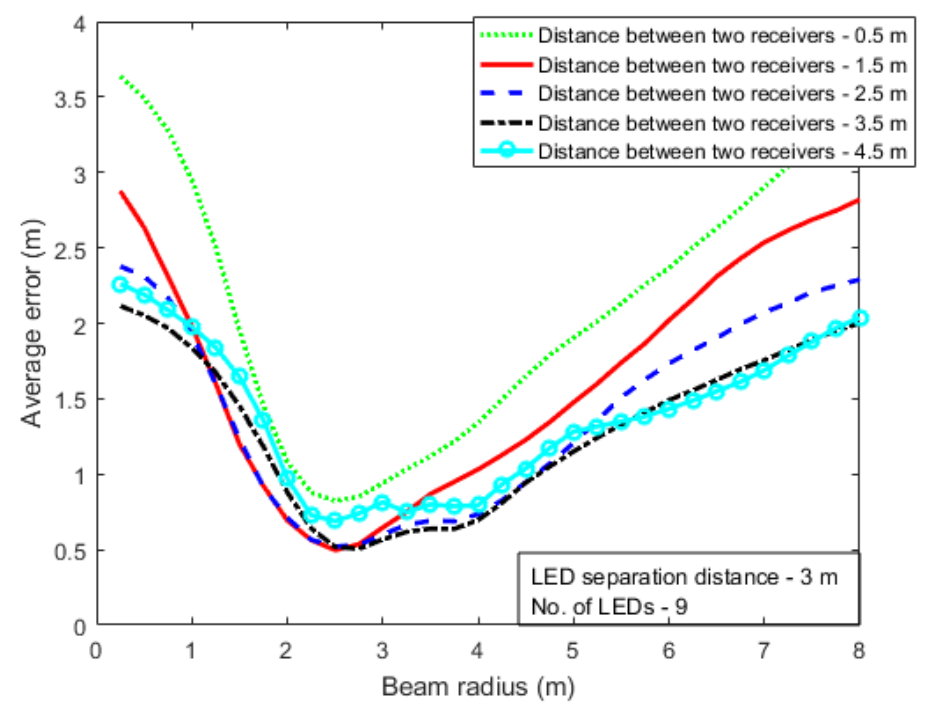

Figure 16. Average error vs. beam radius in a TRIP algorithm for different distances between two receivers.

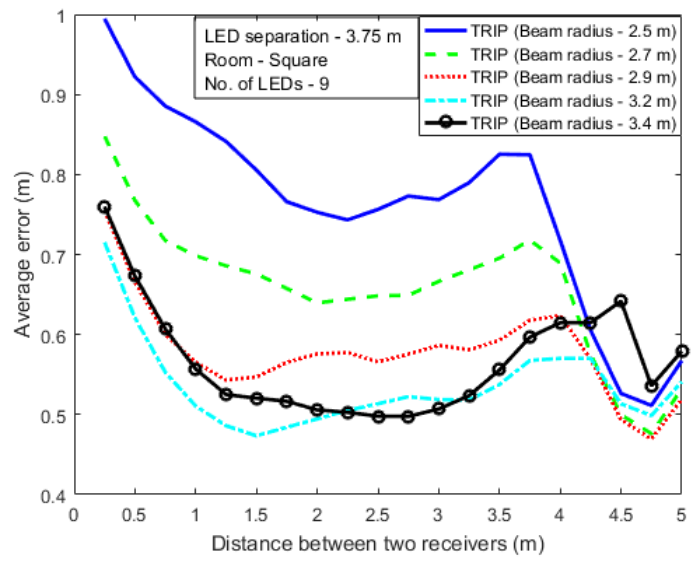

(a)

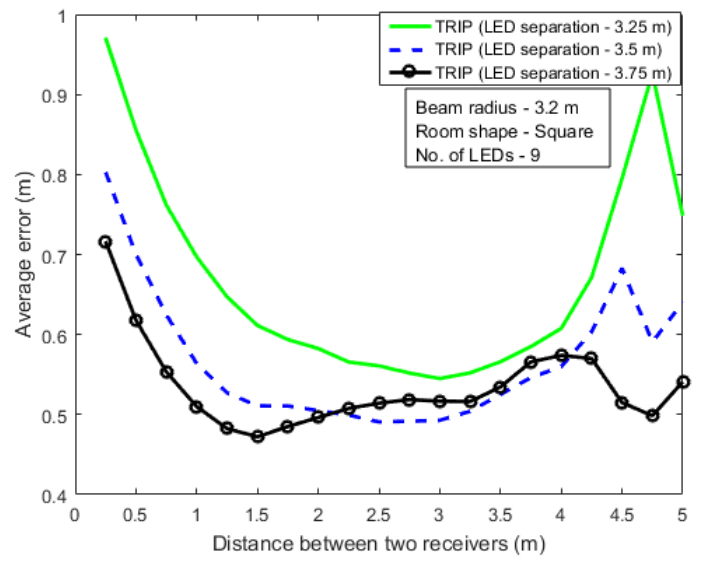

(b)

Figure 17. Average error vs. distance between two receivers in the TRIP algorithm (a) different beam radius; (b) different LED separation distance.

\subsection{Different Beam Shapes of LEDs}

LEDs used for indoor or outdoor lighting purposes have a major disadvantage that the illumination they produce is non-uniform with the centre having high intensity, and the edges having low intensity of illumination. A recent research presented an energy mapping technique for designing a free-form lens assuring uniform distribution of intensity in at least $95 \%$ of the illuminated area. Using this technology, it is possible to obtain LED beam patterns [58] with varying geometrical shapes and uniform distribution of light intensity. Considering the possibility of uniformly distributed multiple shapes for a LED beam, the shape of an LED beam might have some effect in calculation of average error in position estimation. For the purpose of analysis, average error is found for different beam shapes like circle, triangle, square, pentagon and hexagon in both OBRIP and TRIP algorithms while keeping the beam area constant.

From the simulations results, as shown in Figure 18, it is observed that the least minimum average error value is observed in case of a square shape beam for the OBRIP algorithm. For a triangular beam shape, the least minimum average error value is the highest as compared to other beam shapes. This is because, for all the other beam shapes, the covered room area is more inclusive. A similar trend is observed for the TRIP algorithm. 


\subsection{OBRIP and TRIP Algorithms with Previous Locations}

As discussed previously, it is assumed in both the proposed algorithms that, if neither of the receivers received a signal, the centre of the room is assigned as the current location of the receiver. This assumption, though seems logical, but might lead to reduced accuracy when the majority of the positions of the receiver are in the non-coverage region. In this paper, a new approach is proposed in which the previous location of the object is assigned as its current location if the receivers are unable to detect a signal. Simulation results have proven that this approach leads to lowering in the average value of error as the previous location, in most of the cases, is nearest to the actual location as compared to the centre of the room.

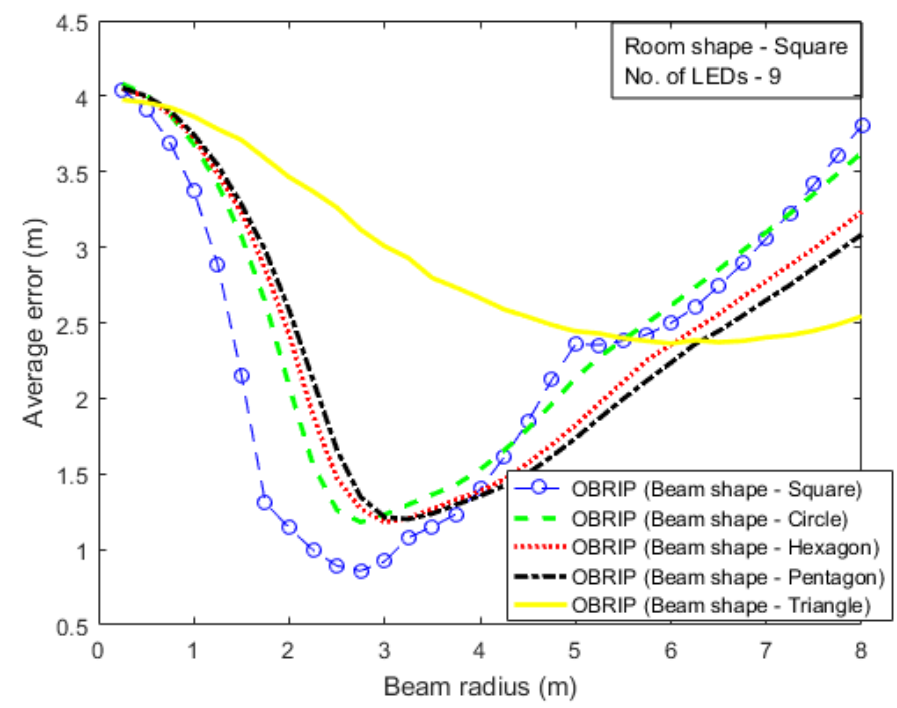

Figure 18. Average error vs. beam radius for different LED beam shapes (OBRIP).

For the performance analysis, for both the OBRIP and TRIP algorithms, 25,000 uniformly distributed random points are generated in the room, with each point $0.5 \mathrm{~m}$ away from the previous generated location coordinate. To the points lying outside the LED coverage, the new approach of assigning previous location coordinates is applied and average error is calculated for the entire system. On comparing the results before and after implementation of this technique, it is observed that the previous location method performs better at smaller values of beam radius with average error decreasing by even $10 \%$ in some cases. After the optimal beam radius value, there is a small difference in the error values as shown in Figure 19. Exhaustive simulation taking multiple values of beam radius and LED separation distance also leads to the same inference. Thus, implementing this approach can help extract a higher accuracy from the IPS.

\subsection{Receiver Blockage in the TRIP Algorithm}

In the TRIP algorithm with two receivers, optical signals from the pre-installed LED array continuously transmitting their location coordinates are used for position estimation. The receivers are installed on each of the shoulders of the person taking the distance between them as $0.5 \mathrm{~m}$. However, there exists a possibility that one of the receivers might get blocked or stop working and receive no signal at all. In this scenario, the blocked receiver leads to decrease in positional estimation accuracy. To overcome this situation, a position prediction method is proposed. 


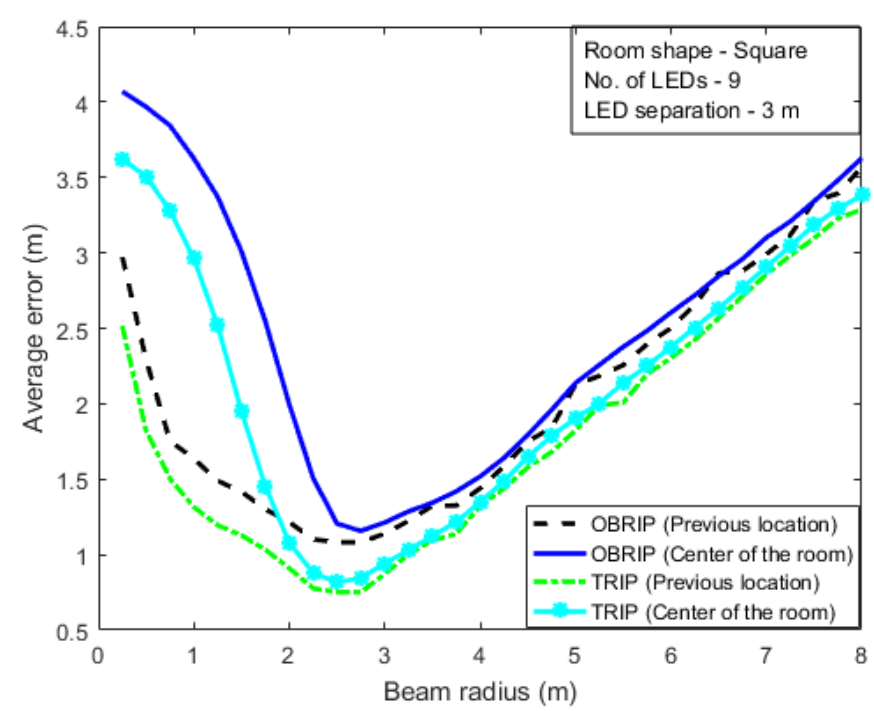

Figure 19. Average error vs. beam radius (OBRIP and TRIP with previous locations).

Consider a situation where one of the two receivers, say $R X_{1}$, is still receiving a signal, but the second receiver, $R X_{2}$, is blocked. In this case, only the reference position of $R X_{1}$ is being calculated and used for position estimation. In the proposed solution, $R X_{2}{ }^{\prime} s$ position is predicted based on $R X_{1}{ }^{\prime} \mathrm{s}$ reference position in such a way that the distance between the $R X_{1}$ 's reference position and predicted $R X_{2}$ position is $0.5 \mathrm{~m}$. Now, as both reference points are available, the average error calculation is performed as before in the TRIP algorithm. From the graph shown in Figure 20, it is observed that the TRIP algorithm with receiver blockage performs better than the OBRIP algorithm leading to a lower value of average error. When comparing its performance to TRIP algorithm without receiver blockage, the average error value difference at smaller and larger radii is almost negligible.

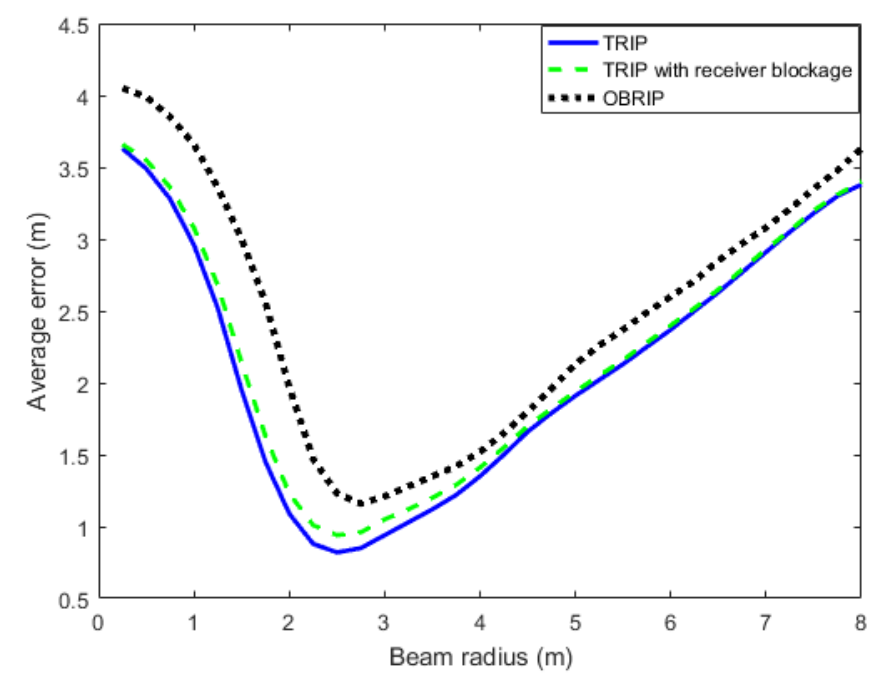

Figure 20. Average error vs. beam radius for OBRIP algorithm, TRIP algorithm and TRIP algorithm with receiver blockage.

\subsection{Room Scaling}

The performance analysis which has been carried out for the proposed algorithms is for a fixed room size with floor dimensions of $10 \mathrm{~m} \times 10 \mathrm{~m}$. There can be an argument that the results might not hold true for rooms of different sizes. This poses a challenge on how to calculate the average error for different sized rooms. Either simulation can be carried out for each and every possible room 
size, or propose a simpler solution where error can be scaled as per the room dimensions relative to a standard room size. The latter option is preferred as it is less complex and time consuming, and simulations are carried out to verify the scaling concept. The fundamental hypothesis is, when the room size is scaled, average error is also scaled by the same factor. This scaling factor is calculated for the new room size based on the dimensions of a standard room size for which the error has already been calculated. A point to note is that room size in this case only refers to length and breadth of the room. Height is not considered while scaling as it depends on the threshold power of the optical receiver, thus it varies as this threshold value changes, making average error independent of the height of the room.

In this research, scaling is performed only for a square room shape with nine LEDs. The standard room size for calculating the scaling factor is taken as $10 \mathrm{~m} \times 10 \mathrm{~m}$. The corresponding average error value for each simulated condition is taken from the previously conducted performance analysis. The ratio of the new room size and the standard room size tells how many times the area of the room has increased or decreased. As area and error have different dimensions, error's dimensions being the square root of dimensions of area, the scaling factor for the average error is the square root of the ratio calculated earlier. This can be better explained with the help of an example. Say, for a standard room size of $10 \mathrm{~m} \times 10 \mathrm{~m}$, the positioning error is estimated to be $1 \mathrm{~m}$. Now, this error value needs to be used for calculating the positioning error for a room of size $5 \mathrm{~m} \times 5 \mathrm{~m}$. The scaling factor in this case is $\sqrt{25 / 100}=0.5$. Thus, for the new room dimension, the average error will be $1 \mathrm{~m} \times 0.5=0.5 \mathrm{~m}$. This result is found to match the average error obtained through simulation for the proposed algorithms as shown in Figure 21. When the dimension of the room is changed from $10 \mathrm{~m} \times 10 \mathrm{~m}$ to $5 \mathrm{~m} \times 5 \mathrm{~m}$, minimum average error and beam radius is scaled by a factor of $1 / 2$. For a room dimension of $2.5 \mathrm{~m} \times 2.5 \mathrm{~m}$, average error and beam radius are scaled by one-fourth with respect to the $10 \mathrm{~m} \times 10 \mathrm{~m}$ room, and by $1 / 2$ with respect to the $5 \mathrm{~m} \times 5 \mathrm{~m}$ room. An important point to note is that the position of the nine LEDs is also scaled by same scaling factor in both the algorithms. In addition, a similar trend is observed for TRIP algorithm.

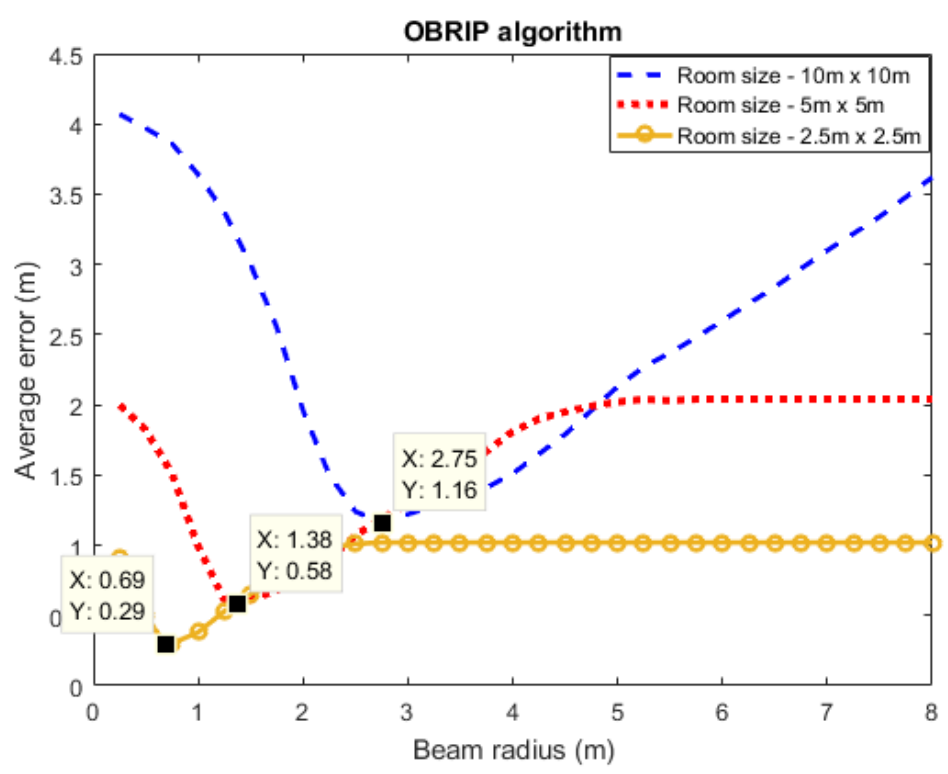

Figure 21. Average error vs. beam radius with room scaling (OBRIP).

\section{Guidelines for Installation}

With the performance of OBRIP and TRIP proving their ability to adapt to various indoor environments and providing accurate position estimation, this section provides guidelines for installation and parameter selection for an OWC-based IPS with the OBRIP or TRIP algorithm: 
1. The first step in the installation process is to determine the size of the room in which the IPS is to be installed. Then, according to the application, the acceptable error in position estimation is chosen. These factors help in determining the value of system parameters.

2. The next step is to determine the number of LEDs to be installed in the room. Referring to Equation (27), if the minimum standard deviation, or error in position estimation, and the room dimensions for a rectangular or square-shaped room are known, the relationship between the number of LEDs corresponding to the length and breadth of the room can be determined.

3. After the number of LEDs are chosen, the position where the LEDs are to be installed on the ceiling of the room needs to be determined. The LED separation distance corresponding to the length and breadth of the room is the ratio of the side length and the number of LEDs along that side.

4. Now, as the LEDs are installed, the next step is to choose the beam radius corresponding to which the desired minimum error can be achieved. This value can be obtained from (28), and the light intensity of the LEDs can be adjusted to obtain this value. A point to note is that this equation is for square and rectangular beam shapes. For a circular beam shape, the results of exhaustive analysis shown in Figure 14a,b can be used to determine the beam radius, depending on upon the chosen algorithm. As these plots are for a room size of $10 \mathrm{~m} \times 10 \mathrm{~m}$, the parameter and error values can be scaled depending upon the room dimensions.

5. The final step for completing the installation in the room is to connect the LEDs to their respective communication systems to enable them to transmit their location information encoded as an optical signal. The transmission module of the OWC-based IPS system is now functional.

6. Now, an optical receiver programmed with an indoor positioning algorithm is attached to the object whose position is to be estimated. Depending on the application and budgetary constraints, the OBRIP or TRIP algorithm is chosen. In the case of the TRIP algorithm, a lower positioning error can be obtained with the same system parameter values by using one extra optical receiver. Based upon the object size, the distance between the receivers is selected. For example, to track very large equipment, the distance between receivers can even be taken as $1.5 \mathrm{~m}$.

\section{Conclusions}

Research on IPS has been gaining steam for the past few years since the success of GPS navigation systems in outdoor environments. The demand for less complex, low cost and highly accurate positioning algorithms for implementation of an IPS has led to the design of the OBRIP and TRIP algorithms. These algorithms take the advantage of the overlapping light beam regions to improve the accuracy of the IPS by generating more reference points for position assignment. This research proves the feasibility of using OBRIP and TRIP algorithms in an indoor environment, and confirms the performance analysis results achieved from the previous analysis, which did not take into account the effect of channel parameters, by producing similar results in the presence of a simulated indoor wireless optical channel in LOS configuration. The TRIP algorithm gives smallest average error value of $0.82 \mathrm{~m}$ against the value of $1.16 \mathrm{~m}$ obtained in the OBRIP algorithm before the optimisation of system parameters. In addition, in this research, the trend of average error variation when different beam shapes are used is showcased. From all the results, it can be concluded that all the shapes, with the exception of triangular beam shape, have similar average error values. Triangular shape, not being able to completely encompass the area of the room, leads to a higher value of average error in position estimation.

Exhaustive simulation performed in this research to find the 90th percentile error values with various combinations of beam radius and LED separation distance gives a highly accurate determination of possible values of error. From the analysis, the 90th percentile error obtained for the TRIP algorithm is $0.94 \mathrm{~m}$ against $1.2 \mathrm{~m}$ obtained from the OBRIP algorithm, which is a $21 \%$ drop in error value. In terms of average error, at optimal values of beam radius and LED separation distance of $3.2 \mathrm{~m}$ and $3.75 \mathrm{~m}$, respectively, the smallest average error value obtained from the TRIP algorithm is 
$0.61 \mathrm{~m}$, whereas, in the OBRIP algorithm at optimal values of $3.4 \mathrm{~m}$ and $4 \mathrm{~m}$ of beam radius and LED separation distance, respectively, the smallest average error value is $0.81 \mathrm{~m}$, thus proving superior performance of TRIP algorithm over OBRIP and achieving an error value, which is $6 \%$ of the smallest room dimension along the floor plane. This level of accuracy is adequate for designing a position tracking system for people with dementia to determine if they are in the vicinity of dangerous objects or situations. In addition, it is observed that, for the TRIP algorithm, these optimal values of beam radius and LED separation can give average error values under $0.5 \mathrm{~m}$ if the distance between two receivers is varied.

This paper also helps to find the relationship between overlapping distance and length of the room for minimum standard deviation for a multiple LED system with rectangular and square beam shapes. It confirms the intuition that minimum error should occur when all the LED light beam regions have the same area. These expressions can be used to calculate system parameter values to obtain minimum position error for a room of any size. Another major contribution of this paper is derivation of the closed-form expression for standard deviation for overlapping circular beam shapes.

This research also provides a range of values for system parameters for which the average error is under $1 \mathrm{~m}$, thus allowing custom selection of the values of these system parameters which are suitable for different room shapes, sizes and designs. These positioning algorithms being low-cost and highly accurate can revolutionize the indoor positioning domain, and lead to development of indoor positioning and tracking systems which can benefit the medical and commercial applications sectors. Some possible applications include tracking physical movement of patients in medical facilities, inventory management in large warehouses, and navigation within museums, airports, railway stations and large shopping malls.

Author Contributions: Formal analysis, M.A.; Investigation, M.A.; Methodology, M.A.; Project administration, S.S.; Supervision, S.S. and T.B.; Writing—original draft, M.A.; Writing—review \& editing, S.S. and T.B.

Funding: This research is funded by Glasgow Caledonian University.

Conflicts of Interest: The authors declare no conflict of interest.

\section{Abbreviations}

The following abbreviations are used in this manuscript:

$\begin{array}{ll}\text { ADOA } & \text { Angle Difference of Arrival } \\ \text { AOA } & \text { Angle of Arrival } \\ \text { CDF } & \text { Cumulative Distribution Frequency } \\ \text { EM } & \text { Electromagnetic Interference } \\ \text { FOV } & \text { Field of Vision } \\ \text { GPS } & \text { Global Positioning System } \\ \text { IPS } & \text { Indoor Positioning System } \\ \text { LED } & \text { Light Emitting Diode } \\ \text { LOS } & \text { Line of Sight } \\ \text { MLEM } & \text { Multiple LED Estimation Model } \\ \text { OBRIP } & \text { Optimal Beam Radius Indoor Positioning } \\ \text { OOK } & \text { On-Off Keying } \\ \text { OWC } & \text { Optical Wireless Communication } \\ \text { PD } & \text { Photo Diode } \\ \text { PDM } & \text { Pulse Duration Multiplexing } \\ \text { RMSE } & \text { Root Mean Square Error } \\ \text { RSS } & \text { Received Signal Strength } \\ \text { TDOA } & \text { Time Difference of Arrival } \\ \text { TOA } & \text { Time of Arrival } \\ \text { TRIP } & \text { Two Receiver Indoor Positioning }\end{array}$




\section{Appendix A}

In this appendix, the derivation of standard deviation is carried out for two overlapping circles forming three separate regions in the shape of a 'crescent' and 'eye' as shown in Figure 6. In order to find the standard deviation for the eye, the second raw moment is derived around the centre of the eye using (A3) and is normalised by the area of the eye as given by (A4):

$$
\begin{gathered}
\mu_{2 e}=\int_{0}^{\frac{\pi}{2}} \int_{0}^{U_{l}} r^{2}(r \mathrm{~d} r \mathrm{~d} \theta), \\
r_{1}^{2}+d^{2}-2 r_{1} d \cos (\pi-\theta)=r^{2}, \\
r_{1}=-d \cos \theta+\sqrt{d^{2}\left(\cos ^{2} \theta-1\right)+r^{2}},
\end{gathered}
$$

where

$$
\begin{gathered}
r_{1}=U_{l}, U_{l}=-d \cos \theta+\sqrt{d^{2}\left(\cos ^{2} \theta-1\right)+r^{2}}, \\
\mu_{2 e}=\frac{1}{4} \int_{0}^{\frac{\pi}{2}}\left(-d \cos \theta+\sqrt{d^{2}\left(\cos ^{2} \theta-1\right)+r^{2}}\right)^{4} \mathrm{~d} \theta \\
=d^{4}\left(c(c+2) \tan ^{-1}(\sqrt{c-1})-3 c \sqrt{c-1}\right),
\end{gathered}
$$

where

$$
\begin{gathered}
c=\frac{r^{2}}{d^{2}}, \\
\sigma_{e}=\sqrt{\frac{\mu_{2 e}}{A_{e}}} .
\end{gathered}
$$

Equation (A5) is used to find the second raw moment of the eye with respect to centre of the circle:

$$
\begin{aligned}
& \mu_{2 e c}=\mu_{2 e}+A_{e} d^{2}, \\
& \mu_{2 c r}=\mu_{2 c}-\mu_{2 e c} .
\end{aligned}
$$

The standard deviation for the crescent shape is obtained using (A7)

$$
\sigma_{2 c r}=\sqrt{\frac{\mu_{2 c}-\mu_{2 e c}}{A_{c}-A_{e}}} .
$$

$A_{e}, \mu_{2 c}$ and $A_{c}$ can be calculated using (15), (17) and (20), respectively.

\section{Appendix B}

In order to find the optimal radius at which minimum average error is obtained, the method of derivatives is applied on the overall standard deviation equation for two rectangular overlapping LED beams as shown in Figure 8, which can be expressed as:

$$
\sigma_{2 r}=\left(\frac{2 E_{1} A_{1}+E_{2} A_{2}}{2 A_{1}+A_{2}}\right)
$$


where

$$
\begin{gathered}
E_{1}=\sqrt{\frac{\left(\frac{h-x}{2}\right)^{2}+b^{2}}{12}}, A_{1}=b\left(\frac{h-x}{2}\right), \\
E_{2}=\sqrt{\frac{x^{2}+b^{2}}{12}}, A_{2}=x b .
\end{gathered}
$$

Substituting the values of the above variables in (A8) leads to (A9):

$$
\sigma_{2 r}=\frac{2 \sqrt{\frac{\left(\frac{h-x}{2}\right)^{2}+b^{2}}{12}} b\left(\frac{h-x}{2}\right)+\sqrt{\frac{x^{2}+b^{2}}{12} x b}}{2 b\left(\frac{h-x}{2}\right)+x b} .
$$

The method of derivatives is applied on (A9), which results in (A10):

$$
\begin{gathered}
\frac{\mathrm{d}}{\mathrm{d} x} \sigma_{2 r}=0, \\
2 \sqrt{x^{2}+b^{2}}-\frac{(h-x)^{2}}{\sqrt{(h-x)^{2}+4 b^{2}}}, \\
+2 \frac{x^{2}}{\sqrt{x^{2}+b^{2}}}-\sqrt{(h-x)^{2}+4 b^{2}}=0, \\
2 R_{1}-R_{2}=\frac{C_{1} R_{2}-C_{2} R_{1}}{R_{1} R_{2}},
\end{gathered}
$$

where

$$
\begin{gathered}
R_{1}=\sqrt{x^{2}+b^{2}}, R_{2}=\sqrt{(h-x)^{2}+4 b^{2}}, \\
C_{1}=2 x^{2}, C_{2}=(h-x)^{2} .
\end{gathered}
$$

Simplification of (A11) gives a relationship between $x$ and $h$ as:

$$
\begin{gathered}
\frac{2\left(x^{2}+b^{2}\right)-2 x^{2}}{(h-x)^{2}+4 b^{2}-(h-x)^{2}}=\sqrt{\frac{x^{2}+b^{2}}{(h-x)^{2}+4 b^{2}}}, \\
3 x^{2}+2 h x-h^{2}=0 \\
x=\frac{h}{3} .
\end{gathered}
$$

Having obtained the relationship between $x$ and $h$ in (A12), the value of $x$ is substituted in (A8) to find the minimum standard deviation value for the two overlapping rectangular beams:

$$
\sigma_{2 r m}=\sqrt{\frac{\frac{h^{2}}{9}+b^{2}}{12}} .
$$




\section{References}

1. Dardari, D.; Closas, P.; Djurić, P.M. Indoor tracking: Theory, methods, and technologies. IEEE Trans. Veh. Technol. 2015, 64, 1263-1278. [CrossRef]

2. Luo, P.; Ghassemlooy, Z.; Le Minh, H.; Khalighi, A.; Zhang, X.; Zhang, M.; Yu, C. Experimental demonstration of an indoor visible light communication positioning system using dual-tone multi-frequency technique. In Proceedings of the IEEE 3rd International Workshop in Optical Wireless Communications (IWOW), Funchal, Portugal, 17 September 2014; pp. 55-59.

3. Yang, S.; Jeong, E.; Han, S. Indoor positioning based on received optical power difference by angle of arrival. Electron. Lett. 2014, 50, 49-51. [CrossRef]

4. Zhou, Z.; Kavehrad, M.; Deng, P. Indoor positioning algorithm using light-emitting diode visible light communications. Opt. Eng. 2012, 51, 085009. [CrossRef]

5. Yang, S.H.; Jung, E.M.; Han, S.K. Indoor location estimation based on LED visible light communication using multiple optical receivers. IEEE Commun. Lett. 2013, 17, 1834-1837. [CrossRef]

6. Kim, H.S.; Kim, D.R.; Yang, S.H.; Son, Y.H.; Han, S.K. An indoor visible light communication positioning system using a RF carrier allocation technique. J. Lightw. Technol. 2013, 31, 134-144. [CrossRef]

7. Yang, S.H.; Han, S.K. VLC based indoor positioning using single-Tx and rotatable single-Rx. In Proceedings of the IEEE 12th International Conference on Optical Internet (COIN), Jeju, Korea, 27-29 August 2014; pp. 1-2.

8. Koyuncu, H.; Yang, S.H. A survey of indoor positioning and object locating systems. IJCSNS Int. J. Comput. Sci. Netw. Secur. 2010, 10, 121-128.

9. De Lausnay, S.; De Strycker, L.; Goemaere, J.P.; Stevens, N.; Nauwelaers, B. Optical CDMA codes for an indoor localization system using VLC. In Proceedings of the IEEE 3rd International Workshop in Optical Wireless Communications (IWOW), Funchal, Portugal, 17 September 2014; pp. 50-54.

10. Yazici, A.; Yayan, U.; Yücel, H. An ultrasonic based indoor positioning system. In Proceedings of the IEEE International Symposium on Innovations in Intelligent Systems and Applications, Istanbul, Turkey, 15-18 June 2011; pp. 585-589.

11. Yamaguchi, S.; Mai, V.V.; Thang, T.C.; Pham, A.T. Design and performance evaluation of VLC indoor positioning system using optical orthogonal codes. In Proceedings of the IEEE Fifth International Conference on Communications and Electronics (ICCE), Danang, Vietnam, 30 July-1 August 2014; pp. 54-59.

12. Huang, T.; Gao, X.; Guo, Y.; Li, S.; Li, Q.; Li, C.; Zhu, H.; Wang, Y. Visible light indoor positioning fashioned with a single tilted optical receiver. In Proceedings of the IEEE 14th International Conference on Optical Communications and Networks (ICOCN), Nanjing, China, 3-5 July 2015; pp. 1-4.

13. Aminikashani, M.; Gu, W.; Kavehrad, M. Indoor positioning with OFDM visible light communications. In Proceedings of the IEEE 13th IEEE Annual Consumer Communications \& Networking Conference (CCNC), Las Vegas, NV, USA, 9-12 January 2016; pp. 505-510.

14. Zhang, W.; Chowdhury, M.S.; Kavehrad, M. Asynchronous indoor positioning system based on visible light communications. Opt. Eng. 2014, 53, 045105. [CrossRef]

15. Zheng, Y.; Zhang, M. Visible light communications-recent progresses and future outlooks. In Proceedings of the IEEE Symposium on Photonics and Optoelectronics, Chengdu, China, 19-21 June 2010; pp. 1-6.

16. Wei, L.; Zhang, H.; Yu, B.; Guan, Y. High-accuracy indoor positioning system based on visible light communication. Opt. Eng. 2015, 54, 110501. [CrossRef]

17. Yang, S.H.; Jeong, E.M.; Kim, D.R.; Kim, H.S.; Son, Y.H.; Han, S.K. Indoor three-dimensional location estimation based on LED visible light communication. Electron. Lett. 2013, 49, 54-56. [CrossRef]

18. Hassan, N.U.; Naeem, A.; Pasha, M.A.; Jadoon, T.; Yuen, C. Indoor positioning using visible LED lights: A survey. ACM Comput. Surv. (CSUR) 2015, 48, 20. [CrossRef]

19. Do, T.H.; Yoo, M. An in-depth survey of visible light communication based positioning systems. Sensors 2016, 16, 678. [CrossRef] [PubMed]

20. Lin, B.; Ghassemlooy, Z.; Lin, C.; Tang, X.; Li, Y.; Zhang, S. An indoor visible light positioning system based on optical camera communications. IEEE Photonics Technol. Lett. 2017, 29, 579-582. [CrossRef]

21. Vatansever, Z.; Brandt-Pearce, M. Visible Light Positioning with Diffusing Lamps Using an Extended Kalman Filter. In Proceedings of the IEEE Wireless Communications and Networking Conference (WCNC), San Francisco, CA, USA, 19-22 March 2017; pp. 1-6. 
22. Dementia-Signs, Symptoms, Cause, Tests, Treatment, Care. Available online: https://www.alz.org/whatis-dementia.asp (accessed on 29 September 2018).

23. Basics of Alzheimer's Disease and Dementia. Available online: https://www.nia.nih.gov/health/whatdementia (accessed on 29 September 2018).

24. Vuong, N.K.; Chan, S.; Lau, C.T. mHealth sensors, techniques, and applications for managing wandering behavior of people with dementia: A review. In Mobile Health; Springer: Berlin, Germany, 2015, Volume 49, pp. 11-42.

25. Protopappas, V.; Tsiouris, K.; Chondrogiorgi, M.; Tsironis, C.; Konitsiotis, S.; Fotiadis, D.I. ALZCARE: An information system for screening, management and tracking of demented patients. In Proceedings of the IEEE 38th Annual International Conference of the IEEE Engineering in Medicine and Biology, Orlando, FL, USA, 16-20 August 2016; pp. 5364-5367.

26. Ajmani, M.; Sinanović, S.; Boutaleb, T. Optimal beam radius for LED-based indoor positioning algorithm. In Proceedings of the IEEE International Conference for Students on Applied Engineering (ICSAE), Newcastle upon Tyne, UK, 20-21 October 2016; pp. 357-361.

27. Ajmani, M.; Boutaleb, T.; Sinanović, S. Optical Wireless Communication based two-receiver Indoor Positioning algorithm. In Proceedings of the IEEE 13th International Wireless Communications and Mobile Computing Conference, Valencia, Spain, 26-30 June 2017; pp. 653-658.

28. Jung, S.Y.; Hann, S.; Park, C.S. TDOA-based optical wireless indoor localization using LED ceiling lamps. IEEE Trans. Consum. Electron. 2011, 57, 1592-1597. [CrossRef]

29. Armstrong, J.; Sekercioglu, Y.A.; Neild, A. Visible light positioning: A roadmap for international standardization. IEEE Commun. Mag. 2013, 51, 68-73. [CrossRef]

30. Zhu, B.; Cheng, J.; Wang, Y.; Yan, J.; Wang, J. Three-dimensional VLC positioning based on angle difference of arrival with arbitrary tilting angle of receiver. IEEE J. Sel. Areas Commun. 2018, 36, 8-22. [CrossRef]

31. Bergen, M.H.; Schaal, F.S.; Klukas, R.; Cheng, J.; Holzman, J.F. Toward the implementation of a universal angle-based optical indoor positioning system. Front. Optoelectron. 2018, 11, 116-127. [CrossRef]

32. Li, Q.L.; Wang, J.Y.; Huang, T.; Wang, Y. Three-dimensional indoor visible light positioning system with a single transmitter and a single tilted receiver. Opt. Eng. 2016, 55, 106103. [CrossRef]

33. Wang, C.; Wang, L.; Chi, X.; Liu, X.; Shi, W; Deng, J. The research of indoor positioning based on visible light communication. China Commun. 2015, 12, 85-92. [CrossRef]

34. Papapostolou, A.; Chaouchi, H. Scene analysis indoor positioning enhancements. Ann. Telecommun. 2011, 66, 519-533. [CrossRef]

35. Xie, C.; Guan, W.; Wu, Y.; Fang, L.; Cai, Y. The LED-ID Detection and Recognition Method Based on Visible Light Positioning Using Proximity Method. IEEE Photonics J. 2018, 10, 1-16. [CrossRef]

36. Luo, J.; Fan, L.; Li, H. Indoor Positioning Systems Based on Visible Light Communication: State of the Art. IEEE Commun. Surv. Tutor. 2017, 19, 2871-2893. [CrossRef]

37. Popoola, O.R.; Sinanović, S. Design and Analysis of Collision Reduction Algorithms for LED-Based Indoor Positioning With Simulation and Experimental Validation. IEEE Access 2018, 6, 10754-10770. [CrossRef]

38. Sertthin, C.; Tsuji, E; Nakagawa, M; Kuwano, S.; Watanabe, K. A switching estimated receiver position scheme for visible light based indoor positioning system. In Proceedings of the IEEE 4th International Symposium on Wireless Pervasive Computing, Melbourne, VIC, Australia, 11-13 February 2009; pp. 1-5.

39. Sertthin, C.; Ohtsuki, T.; Nakagawa, M. 6-axis sensor assisted low complexity high accuracy-visible light communication based indoor positioning system. IEICE Trans. Commun. 2010, 93, 2879-2891. [CrossRef]

40. Sertthin, C.; Fujii, T.; Takyu, O.; Umeda, Y.; Ohtsuki, T. On physical layer simulation model for 6-axis sensor assisted VLC based positioning system. In Proceedings of the IEEE IEEE Global Telecommunications Conference-GLOBECOM, Kathmandu, Nepal, 5-9 December 2011; pp. 1-5.

41. Street, A.; Stavrinou, P.; O’Brien, D.; Edwards, D. Indoor optical wireless systems-A review. Opt. Quantum Electron. 1997, 29, 349-378. [CrossRef]

42. Gao, J.; Yang, F.; Ma, X. Indoor positioning system based on visible light communication with gray-coded identification. In Proceedings of the IEEE 13th International Wireless Communications and Mobile Computing Conference, Valencia, Spain, 26-30 June 2017; pp. 899-903.

43. Popoola, O.; Ogunkoya, F.; Popoola, W.; Ramirez-Iniguez, R.; Sinanović, S. Indoor localization based on Multiple LEDs Position Estimation. In Proceedings of the IEEE 17th International Workshop on Signal Processing Advances in Wireless Communications (SPAWC), Edinburgh, UK, 3-6 July 2016; pp. 1-6. 
44. Li, L.; Hu, P.; Peng, C.; Shen, G.; Zhao, F. Epsilon: A Visible Light Based Positioning System. In Proceedings of the IEEE 11th USENIX Conference on Networked Systems Design and Implementation (NSDI), Seattle, WA, USA, 2-4 April 2014; pp. 331-343.

45. Average Male and Female Dimensions/Heights. Available online: https://www.firstinarchitecture.co.uk/ average-male-and-female-dimensions/ (accessed on 1 October 2018).

46. Ghassemlooy, Z.; Popoola, W.; Rajbhandari, S. Optical Wireless Communications: System and Channel Modelling with MATLAB; CRC Press: Boca Raton, FL, USA, 2012.

47. Kahn, J.M.; Barry, J.R. Wireless infrared communications. Proc. IEEE 1997, 85, 265-298. [CrossRef]

48. Jungnickel, V.; Pohl, V.; Nonnig, S.; Von Helmolt, C. A physical model of the wireless infrared communication channel. IEEE J. Sel. Areas Commun. 2002, 20, 631-640. [CrossRef]

49. Gu, W.; Zhang, W.; Kavehrad, M.; Feng, L. Three-dimensional light positioning algorithm with filtering techniques for indoor environments. Opt. Eng. 2014, 53, 107107. [CrossRef]

50. Hayasaka, N.; Ito, T. Channel modeling of nondirected wireless infrared indoor diffuse link. Electron. Commun. Jpn. (Part I Commun.) 2007, 90, 9-19. [CrossRef]

51. Moment. Available online: http:/ / mathworld.wolfram.com/Moment.html (accessed on 2 October 2018).

52. Raw Moment. Available online: http://mathworld.wolfram.com/RawMoment.html (accessed on 2 October 2018).

53. Disk Point Picking. Available online: http://mathworld.wolfram.com/DiskPointPicking.html (accessed on 2 October 2018).

54. Rogerson, P.A. Statistical Methods for Geography: A Student's Guide; Sage: Newcastle upon Tyne, UK, 2014.

55. Rogerson, P.; Yamada, I. Statistical Detection and Surveillance of Geographic Clusters; CRC Press: Boca Raton, FL, USA, 2008.

56. Stewart, R.; Zhang, H. A note concerning the distances of uniformly distributed points from the centre of a rectangles. Bull. Aust. Math. Soc. 2013, 87, 115-119. [CrossRef]

57. Beer, F.P.; Johnston Jr, E.R.; Mazurek, D.F.; Cornwell, P.J.; Eisenberg, E.R.; Sanghi, S. Vector Mechanics for Engineers; Tata McGraw-Hill Education: New York, NY, USA, 1972; Volume 1.

58. Babadi, S.; Ramirez-Iniguez, R.; Boutaleb, T.; Mallick, T. Analysis of uniformity of illumination of a freeform lens when combined with different optical source. In Proceedings of the SPIE OPTO, San Francisco, CA, USA, 24 February 2016; p. 974417.

(C) 2018 by the authors. Licensee MDPI, Basel, Switzerland. This article is an open access article distributed under the terms and conditions of the Creative Commons Attribution (CC BY) license (http:// creativecommons.org/licenses/by/4.0/). 